\title{
1 Improved Photocleavable Proteins with Faster and More Efficient
}

\section{Dissociation}

3 Xiaocen Lu${ }^{1}$, Yurong Wen²,3,7, Shuce Zhang ${ }^{1,7}$, Wei Zhang ${ }^{1,4}$, Yilun Chen ${ }^{5}$, Yi Shen ${ }^{1}$, M. Joanne

$4 \quad$ Lemieux $^{2}$, and Robert E. Campbell ${ }^{1,6} *$

$5 \quad{ }^{1}$ Department of Chemistry, University of Alberta, Edmonton, Alberta, T6G 2G2, Canada.

$6{ }^{2}$ Department of Biochemistry, Membrane Protein Disease Research Group, University of

7 Alberta, Edmonton, Alberta, T6G 2H7, Canada.

$8{ }^{3}$ Current address: Talent Highland, The First Affiliated Hospital, Xi'an Jiaotong University,

9 Xi'an, Shaanxi, 710061, China.

$10{ }^{4}$ Current address: Department of Chemistry, Stanford University, Stanford, California, 94305,

11 United States.

$12{ }^{5}$ Department of Biochemistry, University of Alberta, Edmonton, Alberta, T6G 2H7, Canada.

$13{ }^{6}$ Department of Chemistry, The University of Tokyo, Tokyo, 113-0033, Japan.

$14 \quad 7$ These authors contributed equally to this work.

15 Correspondence and requests for materials should be addressed to R.E.C. (email:

16 robert.e.campbell@ualberta.ca).

\section{Abstract}

18 The photocleavable protein $(\mathrm{PhoCl})$ is a green-to-red photoconvertible fluorescent protein

19 that, when illuminated with violet light, undergoes main chain cleavage followed by

20 spontaneous dissociation of the resulting fragments. The first generation PhoCl (PhoCl1)

21 exhibited a relative slow rate of dissociation, potentially limiting its utilities for optogenetic

22 control of cell physiology. In this work, we report the X-ray crystal structures of the PhoCl1 
23 green state, red state, and cleaved empty barrel. Using structure-guided engineering and

24 directed evolution, we have developed PhoCl2c with higher contrast ratio and PhoCl2f with

25 faster dissociation. We characterized the performance of these new variants as purified

26 proteins and expressed in cultured cells. Our results demonstrate that PhoCl2 variants

27 exhibit faster and more efficient dissociation, which should enable improved optogenetic

28 manipulations of protein localization and protein-protein interactions in living cells.

\section{Introduction}

30 As a burgeoning range of biological techniques, optogenetics, which involves the use of light and

31 genetically encodable light-sensitive proteins, enables unprecedented levels of control of

32 numerous biological processes ranging from cellular activities to animal behaviours ${ }^{1-3}$.

33 Genetically encodable light-sensitive proteins, as a key component of optogenetic actuators, are

34 typically engineered from natural photoreceptors ${ }^{4}$. The current repertoire of engineered

35 optogenetic actuators can be divided into the following categories ${ }^{4,5}$ : (i) opsin-based light-

36 activatable channels or pumps (e.g., ChR2 [Ref. 6], eNpHR ${ }^{7}, \mathrm{eBR}^{8}$ and OptoXR ${ }^{9}$ ); (ii) proteins

37 with light-induced allosteric change (e.g., LINuS $^{10}$, LEXY $^{11}$ and PA-Rac1 [Ref. 12]); (iii) proteins

38 with light-induced dimerization (e.g., TULIPs ${ }^{13}$, Magnets ${ }^{14}$, CRY2-CIB1 [Ref. 15], PhyB-PIF ${ }^{16}$,

39 BphP1-PpsR2 [Ref. 17] and Dronpa 145N ${ }^{18}$ ); and (iv) photocleavable protein (PhoCl) ${ }^{5}$. As this

40 summary reveals, $\mathrm{PhoCl}$ is the sole member of a distinct class of optogenetic actuators and

41 uniquely enables irreversible optogenetic activation via a mechanism that requires cleavage of a

42 covalent bond.

The first generation PhoCl was engineered from a circularly permuted (cp) green-to-red

44 photoconvertible fluorescent protein (FP) that, when illuminated with $400 \mathrm{~nm}$ violet light,

45 undergoes a main chain-breaking $\beta$-elimination at the green chromophore ${ }^{19}$. The bond cleavage 
46 produces a large $\mathrm{N}$-terminal empty barrel fragment and a small C-terminal peptide fragment which

47 spontaneously dissociate (as schematically represented in Fig. 1a,b). Due to this property, PhoCl1

48 can be used as a relatively simple yet versatile tool for covalent caging of a protein of interest that

49 can be subsequently activated irreversibly by light. We previously demonstrated the use of PhoCl1

50 for the engineering a photoactivatable Cre recombinase, Gal4 transcription factor, and a protease

51 that was used in turn to activate the ion channel Pannexin-1 (PanX1) ${ }^{5}$. Others have further

52 expanded the applications of PhoCl1 to include photo-responsive biomaterials ${ }^{20,21}$, photo-control

53 of cell-to-cell mechanotransduction ${ }^{22}$, and light-induced protein phase separation ${ }^{23}$. Although

54 PhoCl1 has been applied in a growing number of cell physiology and biomaterials applications, a

55 substantial drawback has been the relatively long half-time $\left(t_{1 / 2}\right)$ of fragment dissociation $\left(t_{1 / 2} \sim\right.$

$56 \quad 500 \mathrm{~s})^{5}$.

57 To increase the general utility and applicability of $\mathrm{PhoCl}$, we undertook the development

58 of second generation $\mathrm{PhoCl}$ variants $(\mathrm{PhoCl} 2)$ with improved rate and efficiency of dissociation.

59 To achieve this goal, we first solved the X-ray crystal structures of the green state, the red state,

60 and the cleaved empty barrel of PhoCl1. Then, using structure-guided engineering and directed

61 evolution, in combination with a NanoLuc luciferase ${ }^{24}$-based screening assay ${ }^{25}$, we engineered the

62 PhoCl2c variant with higher dissociation contrast ratio and the PhoCl2f variant with faster

63 dissociation rate. Compared to the original PhoCl1, these PhoCl2 variants exhibit improved

64 dissociation as purified proteins and in cell-based experiments. We further applied $\mathrm{PhoCl} 2 \mathrm{c}$ for

65 the control of light-induced cell apoptosis in living cells. These results establish the improved

66 variants, $\mathrm{PhoCl} 2 \mathrm{c}$ and $\mathrm{PhoCl} 2 \mathrm{f}$, as useful tools for optogenetic control of protein localization and

67 function in live cells.

\section{Results}




\section{Crystal structures of PhoCl1}

70 To gain a better understanding of the structural changes associated with photoconversion and

71 peptide dissociation, and to aid our protein engineering efforts, we determined the X-ray crystal

72 structures of the $\mathrm{PhoCl} 1$ green state $(2.1 \AA$ resolution), red state $(2.3 \AA$ resolution), and the empty

73 barrel fragment (2.8 A resolution) (Fig. 1b and Supplementary Table 1). The structure of the

74 peptide fragment was not determined experimentally and is schematically represented in Fig. 1b

75 only for the sake of clarity.

To produce the PhoCl1 red state crystals, we first crystallized the purified PhoCl1 in its

77 default green state, and then subjected these crystals to in situ partial photoconversion

78 (Supplementary Fig. 1a). By illumination with $405 \mathrm{~nm}$ violet light (15 s light with LED array,

$793.46 \mathrm{~mW} / \mathrm{mm}^{2}$ ), green state PhoCl1 crystals changed from a visibly yellow color to a visibly

80 orange-red color, indicating successful photoconversion. The visibly orange-red color of the

81 photoconverted PhoCl1 appeared to be indefinitely stable as it was maintained for at least one

82 month. We did not observe color loss in the context of the photoconverted red state crystal,

83 suggesting no peptide dissociation occurred, presumably due to the very high concentration of

84 protein and/or steric constraints imposed by crystal packing.

To purify the $\mathrm{PhoCl}$ empty barrel protein for crystallization, the intact $\mathrm{PhoCl}$ protein with

86 a C-terminal $6 \times$ His purification tag was captured on a column of Ni-NTA agarose beads and then

87 photoconverted on the column with repeated violet light illumination (405 nm LED array, 3.46

$88 \mathrm{~mW} / \mathrm{mm}^{2}$ ) to induce photocleavage and dissociation. The eluent containing the PhoCl1 empty

89 barrel was collected, further purified by gel filtration chromatography, and then the protein was

90 crystallized. 
The green and red state structures revealed the changes in the chromophore structure

92 associated with photoconversion. The green chromophore of PhoCl1 is generated from the

93 autogenic post-translational modification of His232, Tyr233, and Gly234 (residues numbering are

94 consistent with the numbering used in the X-ray crystal structure of the green state PhoCl1), which

95 are structurally aligned with Ser65, Tyr66, and Gly67, respectively, of the canonical Aequorea

96 victoria green FP (GFP; PDB ID: 1EMA) ${ }^{26,27}$. Determination of the protein structure using the

97 partially photoconverted crystal revealed a mixture of green and red PhoCl1 states. As expected

98 on the basis of the known photoconversion mechanism ${ }^{19,28,29}$, the red state of PhoCl1 was cleaved

99 at the amide bond connecting Phe231 to His232 (Fig. 1c). Other than the changes in the vicinity

100 of Phe231 and the chromophore, the overall structures of the green and red states are essentially

101 identical.

102

To our surprise, the best fit of the electron density obtained from the partially

103 photoconverted crystal was achieved by modeling only 2 of the 6 monomers in the asymmetric

104 unit in the red state. Initial attempts to model all of the 6 monomers in the asymmetric unit as a

105 mixture of green and red chromophore structures resulted in unsatisfactory fits to the experimental

106 electron density. The best fit of the electron density was achieved by modeling 2 monomers as red

107 state chromophores (chains A and B), and 4 monomers as green state chromophores (chains C, D,

108 E, F). It remains unclear why 2 of 6 monomers were preferentially photoconverted upon

109 illumination, rather than all 6 monomers experiencing equal photoconversion and each existing as

110 a mixture of $33 \%$ red state and $67 \%$ green state. We speculate that efficient Förster resonance

111 energy transfer (FRET) between the closely packed proteins in the crystal may have funneled

112 energy into those monomers that were in the least favorable orientation to act as FRET donors

113 and/or the most favorable orientation to act as FRET acceptors. These monomers may have been 
more likely to undergo photoconversion due to their higher probability of excitation or longer

115 excited state lifetimes.

The structure of the PhoCl1 empty barrel revealed substantial conformational changes

117 relative to the red state, as expected given the complete absence of the C-terminal fragment.

118 Photocleavage and dissociation leaves a water-filled cavity where the peptide fragment previously

119 resided in the interior of the empty barrel. To better visualize the conformational changes between

120 PhoCl1 red state and the empty barrel, the root-mean-square deviation (RMSD) for all residues

121 (that is, the average RMSD for all atoms in each residue) between the two structures was calculated,

122 and the resulting RMSD values were used to color the cartoon coil alignment representation and

123 adjust the coil diameter (thicker coil indicates higher RMSD) (Fig. 1d). This representation

124 revealed that the most substantial, and observable, conformational changes occur in the 201-207

125 loop (highlighted in Fig. 1d). Specifically, in the empty barrel structure, the 201-207 loop region

126 is "folded in" towards the center of the barrel, relative to its position in the red state structure (Fig.

127 1e). Due to this reorientation of the 201-207 loop into the barrel, the cavity in the empty barrel

128 structure differs in shape from the volume that had been occupied by the C-terminal peptide

129 (Supplementary Fig. 1b,c). We speculate that this conformational change helps to stabilize the

130 dissociated empty barrel structure by partially filling the hydrophobic void generated by

131 dissociation of the small peptide fragment. Accordingly, we reasoned that introducing mutations

132 within or near the 201-207 loop region may affect the rate and efficiency of peptide dissociation,

133 and we therefore focussed our efforts on this key region in our subsequent engineering.

\section{Molecular dynamics (MD) simulation of the dissociation process}

135 To gain insight into the dissociation process of PhoCl1 at the molecular level, an adaptive steered 136 molecular dynamics (ASMD) scheme (Supplementary Fig. 2a) was employed to investigate the 
137 dissociation pathway. Overall, 3 structures with distinct conformations of the modeled cp linker

138 (i.e., the linker that connects the original C- and N-termini, Supplementary Fig. 2b) served as the

139 initial coordinates of the ASMD, all of which were taken from a 210 ns unstrained canonical

140 molecular dynamics (cMD) trajectory during which no spontaneous dissociation occurred

141 (Supplementary Fig. 2a,b and Supplementary Movie 1-4). During the ASMD, the distance

142 between the centres-of-mass of the N-terminal barrel and the C-terminal peptide were increased

143 by $1 \AA$ over $1 \mathrm{~ns}$ for each of 40 iterative stages, to give a total final displacement of $40 \AA$. Each

144 stage was repeated 100 times from which an averaged potential mean of force was calculated and

145 the replicate closest to the average was taken as the starting point for the next stage. We simulated

146 the dissociation through 40 such stages without manually specifying the route or direction of

147 dissociation (Supplementary Fig. 2). In all 3 distinct ASMD replications, the C-terminal peptide

148 dissociated through the space between the flexible cp linker (residue 147-172) and the 201-207 loop

149 (Fig. 2a,b and Supplementary Movies 2-4).

Analysis of the RMSD values for all residues over time, with the initial stage as the

151 reference, revealed that the C-terminal peptide showed the highest RMSD due to its physical

152 displacement (Fig. 2c). The region with the second highest RMSD was the 201-207 loop,

153 consistent with conformational changes observed in the experimental crystal structures. Inspection

154 of the dynamic conformational changes during stages of dissociation reveal that the 201-207 loop

155 "flips" out of the barrel, presumably to minimize steric interactions with the C-terminal peptide as

156 it dissociates (Fig. 2b). In contrast, such flipping behaviour of the 201-207 loop was not observed

157 during 210 ns of unrestrained cMD of the red state (Fig. 2d). These results demonstrate that the

158 201-207 loop undergoes a dissociation-dependent conformational change, rather than being

159 intrinsically flexible, providing support for the conclusion that the 201-207 loop would be the key

160 region of interest in our subsequent engineering. 


\section{Engineering and characterization of PhoCl2 variants}

162 In an effort to further improve the utility of $\mathrm{PhoCl}$, we used a combination of structure-guided

163 rational engineering and directed evolution starting from the template of PhoCl1. We first

164 established a NanoLuc luciferase ${ }^{24}$-based assay ${ }^{25}$ for the screening of PhoCl variants libraries on

165 the basis of dissociation rate and efficiency (i.e., extent of dissociation) (Fig. 3a). PhoCl with long

166 flexible linkers at both the $\mathrm{N}$ - and $\mathrm{C}$ - termini was incorporated into the NanoLuc Binary

167 Technology (NanoBiT) system ${ }^{25}$. We found that, upon photoconversion of PhoCl with 30 s of

168 illumination by a $405 \mathrm{~nm}$ LED array, dissociation could be monitored by the decrease in 169 bioluminescence intensity associated with PhoCl-mediated dissociation of NanoBiT into its Large

170 BiT (LgBiT; 18kDa) and Small BiT (SmBiT; 11 amino acid peptide) fragments. To increase the

171 solubility of the SmBiT fragment (fused to the small peptide fragment of PhoCl), we also fused

172 the maltose binding protein (MBP) to the C-terminus of the construct. Using this assay, we

173 observed a $-39 \%$ change in bioluminescence at $460 \mathrm{~nm}$ for PhoCl1 upon illumination

174 (Supplementary Fig. 3).

175 Photoconvertible protein $\mathrm{mMaple}^{30}$ (the progenitor of $\mathrm{PhoCl}$ ) inserted into NanoBiT was

176 used as a negative control for this assay. When using this negative control, we also observed a

177 substantial decrease in apparent bioluminescence $(-26 \%$ at $460 \mathrm{~nm})$. We rationalize this decreased

178 bioluminescence as a result of the change in Bioluminescence Resonance Energy Transfer ${ }^{31}$

179 (BRET) efficiency as the chromophore is converted from green to red. More specifically, BRET

180 to the green state results in sensitized emission that overlaps with the emission of NanoLuc itself.

181 BRET to the red state results in sensitized emission at a longer wavelength peak that does not

182 overlap with that of NanoLuc. This explanation is consistent with the emerging bioluminescent

183 peak at $583 \mathrm{~nm}$ corresponding to the mMaple red state (Supplementary Fig. 3b). 
Starting from the template encoding PhoCl1, two error-prone PCR libraries and 32 site-

185

186

187

188

189

190

191

192

193

194

195

196

197

198

199

200

201

202

203

204

205

206

directed mutagenesis libraries were created. The residues involved in either hydrogen bonding or hydrophobic contacts with the chromophore and dissociable peptide, including the residues of the 201-207 loop (Ile3, Asp5, Phe7, Lys8, Phe11, Gly14, Tyr15, Ile35, Met37, Glu38, Gly39, Asp40, Phe42, Arg77, Asp78, Gly79, Val80, Met87, Tyr117, His118, Val120, His122, Leu138, Tyr139, Val143, Ala144, Arg145, Asn146, Met162, Asp177, Met178, Gly201, Lys202, Pro203, Phe204, Glu205, Gly206, Ile207, Gln208, Ile210, Thr229, Ala230, Thr239 and Lys240) (Supplementary

Fig. 4 and Supplementary Table 2), were randomised by site-directed mutagenesis to introduce a codon (NNK) encoding all 20 amino acids into the corresponding position of the gene. We did not include the residues that are directly hydrogen bonded to the chromophore, in order to avoid disruption of chromophore formation. Green fluorescent clones were picked from libraries expressed in colonies of Escherichia coli on Petri dishes, and bacteria were cultured overnight in small volumes of liquid culture, and the bioluminescence assay was applied in the context of the cell culture lysate. The top $\sim 2 \%$ variants exhibiting large bioluminescence intensity decreases within 5 min after photoconversion were picked for DNA sequencing and also served as templates for subsequent engineering. We observed that most colonies from the site-directed mutagenesis libraries exhibited decreased green fluorescence, likely due to decreased efficiency of chromophore formation. We reasoned that poor chromophore formation was undesirable because proteins without proper chromophore cannot be photoconverted and undergo dissociation, and so we biased our picking towards brighter green fluorescent clones. Notably, this approach may have missed variants that exhibited reduced green fluorescence due to a reduced quantum yield of fluorescence and that may have retained efficient chromophore formation and photocleavage capability. 
Following extensive screening and directed evolution using the bioluminescence assay, we identified a number of specific mutations (Val143Met, Ala144Val, Lys202Leu and Ile207Ser) in or near the 201-207 loop that seemed to be associated with faster dissociation kinetics (Fig. 3b,c,

210 Supplementary Figs. 5, 6, 7, and Supplementary Table 3). To investigate the dissociation

211 kinetics in greater detail, we turned to our previously reported assay that relies on the loss of red

212 fluorescence after photoconversion ${ }^{5}$. The red fluorescence intensity is monitored immediately

213 following $15 \mathrm{~s}$ illumination with $405 \mathrm{~nm}$ light (LED array, $3.46 \mathrm{~mW} / \mathrm{mm}^{2}$ ) (Fig. 3b,c and

214 Supplementary Fig. 8a,b). Consistent with our bioluminescence screening assay, we found that 215 variants with mutations in or near the 201-207 loop (PhoCl1.5f-MV, PhoCl1.5f-L, and PhoCl2f), 216 exhibited faster dissociation rates $\left(t_{1 / 2}=76-140 \mathrm{~s}\right)$ than PhoCl1 $\left(t_{1 / 2}=570 \mathrm{~s}\right)$ (Supplementary

217 Table 4). We next combined these key mutations to explore whether they might have synergetic 218 beneficial effects on the rate of dissociation (Fig. 3c and Supplementary Fig. 8b). Testing a 219 variety of combinations led to the PhoCl1.5f-MVS variant (with Val143Met, Ala144Val and 220 Ile207Ser), the PhoCl1.5f-LS variant (with Lys202Leu and Ile207Ser), and the PhoCl1.5f-MVLS

221 variant (with all four key mutations), all of which exhibited substantially faster dissociation rates $222\left(t_{1 / 2}=15-30 \mathrm{~s}\right)$. The PhoCl1.5f-MVL variant (Fig. 3c), with a combination of Val143Met, 223 Ala144Val and Lys202Leu (without Ile207Ser), has unexpectedly poor dissociation efficiency 224 (with $\sim 60 \%$ red fluorescence remaining as shown in Fig. 3c), illustrating the delicate nature the 225 dissociation process and the importance of empirical screening. All the variants with combined 226 mutations were found to have relatively dim fluorescence brightness (in Supplementary Table 4) 227 possibly due to a decreased efficiency of chromophore formation. The PhoCl1.5f-MVLS variant 228 also has particularly poor expression, possibly indicative a poor folding efficiency, and thus we 229 were unable to purify sufficient protein for a full characterization. We ultimately determined that 
230 the variant with mutations Lys49Gln, Tyr117Cys, Arg145Lys, Asn146Thr and Ile207Ser

231 (designated as PhoCl2f; $t_{1 / 2}=76 \mathrm{~s}$ ) represented the best compromise of dissociation rate,

232 fluorescence brightness, and protein expression level.

233 In parallel to the engineering of faster-dissociating variants, we also identified $\mathrm{PhoCl} 2 \mathrm{c}$

234 (with mutations Lys49Gln, Cys99Gly, Tyr117Cys, Arg145Lys and Asn146Thr) with enhanced

235 dissociation efficiency. To characterize the dissociation efficiency of new PhoCl variants, we used

236 a modified version of our NanoLuc-based bioluminescence screening assay in which we compared

237 the bioluminescence emission spectrum before illumination and 30 minutes after illumination

238 (Supplementary Fig. 3 and Supplementary Table 5). Most PhoCl2 variants exhibited substantial

239 decreases in bioluminescence intensity after illumination, consistent with our expectations

240 (Supplementary Fig. 3a). PhoCl2c and PhoCl1.5f-MVS variants showed the largest decrease

241 around $60 \%$, which indicates that these 2 variants have the best dissociation efficiency. To

242 investigate the dissociation efficiency in greater detail with an orthogonal method, we turned to

243 gel filtration chromatography (GFC) assay combined with analysis of the eluted fractions by SDS-

244 PAGE analysis (Fig. 3d,e and Supplementary Fig. 8c). This analysis demonstrated that the

245 PhoCl2c-MBP fusion had higher dissociation efficiency with less remaining intact protein $(7.9 \%$

246 intact protein) than the PhoCl1-MBP fusion (28.9\% intact protein). Even with the molecular

247 structure of PhoCl1 in hand, it remains unclear to us why PhoCl2c exhibits higher dissociation

248 efficiency. For example, one of the key mutations of PhoCl2c, Cys99Gly, is located on the opposite

249 end of the barrel from the dissociable peptide. PhoCl1.5f-MVS variant has a similarly high

250 dissociation efficiency (7.3\% intact protein) as $\mathrm{PhoCl} 2 \mathrm{c}$, consistent with the bioluminescence

251 analysis, but again the relatively poor chromophore formation of PhoCl1.5f-MVS will likely limit 
252 its further application. Taken together, we successfully engineered two second-generation PhoCl

253 variants: $\mathrm{PhoCl} 2 \mathrm{c}$ with higher dissociation contrast ratio, and $\mathrm{PhoCl} 2 \mathrm{f}$ with faster dissociation rate.

254 Optogenetic control of protein localization by PhoCl2 variants in HeLa cells.

255 The dissociation rate and efficiency of the $\mathrm{PhoCl} 2$ variants was further characterized in transiently

256 transfected HeLa cells using our previously reported assay for manipulating subcellular protein

257 localization through PhoCl-dependent cleavage of a nuclear exclusion sequence (NES) ${ }^{5,32}($ Fig.

258 4a). Photocleavage leads to redistribution of a red FP (mCherry) between the cytoplasm and

259 nucleus. Relative to PhoCl1-mCherry, PhoCl2c-mCherry fusion exhibited a more even red

260 fluorescence redistribution (between the cytoplasm and nucleus) following photocleavage to

261 remove the NES (Fig. 4b). Following protein redistribution after photocleavage, the cytoplasm-

262 to-nucleus intensity ratio for PhoCl2c-mCherry is lower than the ratio for PhoCl1-mCherry (Fig.

$2634 c)$.

The kinetics of photoconversion was determined by loss of green fluorescence, and

265 dissociation was characterized by red fluorescence redistribution in HeLa cells expressing NES-

266 PhoCl-mCherry (Fig. 4d and Supplementary Table 6). PhoCl1.5f-MV, PhoCl1.5f-L and PhoCl2f

267 exhibited faster mCherry redistribution and similar dissociation efficiency (in Supplementary Fig.

268 9) compared to PhoCl1, which is consistent with our in vitro protein characterization results.

269 However, we did find that three other 1.5f variants (PhoCl1.5f-MVS, PhoCl1.5f-LS and

270 PhoCl1.5f-MVLS) did not dissociate as efficiently in cells (refer to Supplementary Fig. 9) as

271 expected, possibly due to poor efficiency of chromophore formation. These results collectively

272 establish the improved characteristics of $\mathrm{PhoCl} 2 \mathrm{c}$ and $\mathrm{PhoCl} 2 \mathrm{f}$ in cellular assays for optogenetic

273 control.

\section{Optogenetic manipulation of cell apoptosis using PhoCl2c}


275 We next attempted to explore possible optogenetic manipulation of cell apoptosis through the use

276 of photocleavage to activate $\mathrm{BH} 3$ interacting-domain death agonist (Bid). Bid is a pro-apoptotic

277 protein belonged to $\mathrm{Bcl}-2$ family. Following cleavage by caspase- 8 during apoptosis, Bid

278 translocates to the mitochondria, where it then induces an increase in outer mitochondria

279 membrane permeability and cytochrome c release ${ }^{33,34}$. To achieve the optogenetic control of

280 apoptosis, we engineered a photoactivatable Bid by inserting PhoCl into the middle of Bid at the

281 position of the caspase-8 cut site (LQTDG) ${ }^{35}$. We reasoned that photoinduced dissociation of C-

282 terminal domain (CBid) from its autoinhibitory N-terminal domain (NBid) would induce the

283 downstream apoptosis pathway (Fig. 5a). We monitored the downstream caspase-3 activity using

284 a NES-DEVD-mCardinal-NLS reporter. The cleavage of DEVD linker of the fusion protein by

285 caspase-3 activation results in the translocation of red FP mCardinal into the nucleus (Fig. 5b).

286 Caspase-3 activation tended to be observed at around $30 \mathrm{~min}$ after conversion for the NBid-

287 PhoCl2c-CBid and around $50 \mathrm{~min}$ after conversion for NBid-PhoCl1-CBid (Fig. 5c,

288 Supplementary Fig. 10a,b and Supplementary Movies 5-7). Cell shrinkage and rounding

289 followed by FP translocation were observed at the end of 2 hours of imaging photoconverted cells.

290 We used the non-dissociable mMaple insertion construct as negative control as it is not expected

291 to dissociate and induce FP translocation following illumination (Fig. 5d). Cells expressing NBid-

292 mMaple-CBid or NBid-PhoCl-CBid variants were illuminated and then cells were analyzed using

293 the DEAD cell viability assay (shown in Fig. 5e and Supplementary Fig. 10c-e). The dye in

294 DEAD stain is ethidium homodimer-1 which is used to indicate loss of cell plasma membrane

295 integrity. For cells expressing NBid-mMaple-CBid, no significant illumination-dependent

296 difference in cell viability was observed. In contrast, illumination of cells expressing NBid-

297 PhoCl1-CBid exhibited significantly higher cell death ratios than non-illuminated cells. A further 
298 enhanced death rate was observed with $\mathrm{PhoCl} 2 \mathrm{c}$ due to its higher dissociation efficiency.

299 Treatment with the cell permeable pan-caspase inhibitor Z-VAD-FMK ${ }^{36}$ effectively reversed this

300 effect, illustrating that cell death was indeed due to caspase activation during apoptosis (shown in

301 Supplementary Fig. 10d,e). In summary, we have designed a novel PhoCl-based optogenetic tool

302 for induction of cell apoptosis pathway. Compared to NBid-PhoCl1-CBid, NBid-PhoCl2c-CBid

303 exhibited more efficient light-dependent induction of apoptosis, providing support for the

304 conclusion that the improved properties (as characterized with purified protein) have been retained

305 for the protein expressed in mammalian cells.

306 Discussion

307 In this work we have described the development of two improved versions of the $\mathrm{PhoCl}$

308 photocleavable protein: the PhoCl2c variant with enhanced dissociation efficiency, and the

309 PhoCl2f variant with faster dissociation rate. This advance was made possible by the determination

310 of the X-ray crystal structure of the empty barrel fragment which revealed substantial

311 conformational changes in the 201-207 loop. During library screening, we discovered four key

312 mutations found within or near 201-207 loop which had a substantial and favorable effect on the

313 PhoCl dissociation rate: Lys202Leu and Ile207Ser in the loop and the combination of Val143Met

314 and Ala144Val on the adjacent $\beta$-strand 11 (Supplementary Fig. 7). Overall, these mutations are

315 clustered in close vicinity to where the C-terminal peptide emerges from the intact protein. It is

316 reasonable to postulated that mutations in this region would affect the efficiency and rate of peptide

317 dissociation. At this point the mechanisms by which these mutations are exerting their observed

318 influence on peptide dissociation is unclear. Two possible mechanisms are: 1) Destabilization of

319 the red state, leading to a decreased energy barrier for peptide dissociation and faster kinetics; and 
2) Increased stabilization of the empty barrel which would likely have little effect on the kinetics, but may lead to a greater overall efficiency of dissociation.

The critical importance of mutations in the loop region was made apparent by the

323 bioluminescence assay which monitored the rate of dissociation. However, this same assay did

324 reveal some unexpected differences in BRET efficiency between different PhoCl variants (refer to

325 Supplementary Fig. 3b and Supplementary Table 5). As with FRET ${ }^{37}$, BRET efficiency

326 strongly depends on the interchromophore distance and orientation. With the same luciferase donor

327 and linkers, the BRET ratio is also related to extinction coefficient and quantum yield of $\mathrm{PhoCl}$

328 acceptor. After photoconversion, the BRET signal should decrease or even disappear as a result of

329 the photocleavage of the green chromophore. Compared to PhoCl1, most of the variants showed

330 decreased BRET efficiencies (that is, higher ratios of NanoLuc emission at $460 \mathrm{~nm}$ to $\mathrm{PhoCl}$

331 emission at $505 \mathrm{~nm}$ ), which can be explained by the diminished extinction coefficients and reduced

332 quantum yields. For these variants with low BRET efficiency, bioluminescence intensity at 460

$333 \mathrm{~nm}$ decreased upon illumination and photocleavage, as expected. However, for reasons that remain

334 unclear to us, PhoCl1.5f-MV and PhoCl1.5f-L exhibited substantially higher BRET efficiency

335 than other variants. Moreover, the luminescence of these 2 variants increased after

336 photoconversion which is contrary to what we observed with the other variants. We speculate that

337 Lys202Leu in PhoCl1.5f-L, and the combination of Val143Met and Ala144Val in PhoCl1.5f-MV,

338 may have introduced stabilizing interactions between NanoLuc and $\mathrm{PhoCl}$ that resulted in a more

339 favorable distance or orientation for increased BRET efficiency. The high BRET ratio still existed

340 in partial converted protein fusion after illumination. Hence, the luminescence signal at $460 \mathrm{~nm}$

341 was observed to increase as a result of the reduced BRET efficiency. 
An important factor that is likely contributing to the improved performance of all of the new variants reported in this work are the increased extinction coefficients at $405 \mathrm{~nm}$

344 (Supplementary Table 4 and Supplementary Fig. 11), which would be expected to lead to more 345 efficient absorption of $405 \mathrm{~nm}$ light and more efficient photoconversion. This higher absorbance at $405 \mathrm{~nm}$ may help to explain why the PhoCl2f and PhoCl1.5f variants, which were selected based

347 due to their faster kinetics of dissociation, also exhibited higher dissociation efficiency than 348 PhoCl1 by GFC analysis (Fig. 3d,e and Supplementary Fig. 8c). In our mammalian cell-based assays, the PhoCl2c constructs exhibited faster redistribution (Fig. 4d and Supplementary Table demonstrated that PhoCl2c was slightly slower at dissociating than PhoCl1 (Fig. 3b). However,

354 due to higher absorbance of $405 \mathrm{~nm}$ light. This result indicates that both the efficiency of 355 photoconversion, and the kinetics of dissociation, are contributing to the effective rate of release 356 in cells.

To summarize, we described the structure-guided engineering of the second generation of

358 PhoCl variants (PhoCl2) with improved dissociation extent (PhoCl2c) and rate (PhoCl2f) by using

359 the luciferase-based screening assay. We expect that this high-throughput screening method could 360 potentially be used to engineer other improved photosensory domains in optogenetic actuators that 361 undergo light-induced heterodimer to monomer transitions (e.g., LOVTRAP ${ }^{38}$ and PhyB-PIF ${ }^{16}$ ).

362 Finally, we anticipate that $\mathrm{PhoCl} 2$ variants will prove useful to in an increased range of optogenetic 363 applications due to their faster and more efficient photo-induced cleavage and dissociation. 


\section{Methods}

\section{General methods and materials.}

All synthetic DNA oligonucleotides were purchased from Integrated DNA Technologies (IDT). Primer sequences are provided in Supplementary Table 7. Plasmid constructions were performed with standard restriction enzyme cloning or In-fusion HD Cloning kit (Takara Bio USA) according to the manufacturer's protocol. Q5 high-fidelity DNA polymerase (New England

371 BioLabs) was used for standard polymerase chain reaction (PCR) amplification, and Taq DNA

372 polymerase (New England BioLabs) was used for error-prone PCR. Restriction enzymes and T4

373 ligase were purchased from Thermo Fisher Scientific. Site-directed mutagenesis was performed 374 using QuikChange Lightening kit (Agilent). Transformation of bacterial cells with plasmid DNA 375 was performed by electroporation using a MicroPulser Electroporator (Bio-Rad). GeneJET gel extraction kit and plasmid miniprep kit (Thermo Fisher Scientific) were used for DNA extraction. DNA sequencing was performed by the University of Alberta Molecular Biology Service Unit.

\section{Library constructions, mutagenesis and screening.}

For constructing the $\mathrm{PhoCl}$ screening vector, the NanoBiT template was ordered as a synthetic gBlock from IDT. The NanoBiT gene sequence was generated using Nluc gene (GenBank: JQ437370.1) as the template, and combining the amino acid substitutions reported in

382 literature ${ }^{25}$. A gene encoding LgBiT-PhoCl-SmBiT containing 5' KpnI and 3' XbaI restriction sites

383 at the ends of $\mathrm{PhoCl}$ was made by overlap extension PCR. Each terminus of $\mathrm{PhoCl}$ and split

384 NanoBiT fragments were joined by linker sequences reported in a previous study ${ }^{25}$. The gene 385 fragments encoding LgBiT-PhoCl-SmBiT (with 5' XhoI and 3' EcoRI restriction sites) and maltose binding protein (MBP) (with 5' EcoRI and 3' HindIII restriction sites) were digested and

387 inserted between the XhoI and HindIII sites of $\mathrm{pBAD} / \mathrm{HisB}$ vector by three-way ligation thus 
yielding the PhoCl screening construct of XhoI-LgBiT-KpnI-PhoCl-XbaI-SmBiT-EcoRI-MBP-

HindIII.

The PhoCl libraries were generated by error-prone PCR or site-directed mutagenesis with

391 degenerate codons at the targeted sites. E. coli strain DH10B (Thermo Fisher Scientific)

392 electrocompetent cells were transformed with the gene libraries and grown on lysogeny broth (LB)

393 agar plates supplemented with $100 \mu \mathrm{g} / \mathrm{mL}$ ampicillin and $0.02 \%$ L-arabinose. For libraries

394 generated by error-prone PCR, approximately 10,000 colonies were screened in a given round.

395 The colonies exhibiting the brightest green fluorescence (top 2\%), as determined using a custom-

396 built colony screener $^{5}$ (for green channel: 470/40 nm excitation and 510/20 nm emission), were

397 picked and cultured in $1 \mathrm{~mL}$ medium in 96-well deep block plates (Thermo Fisher Scientific). For

398 libraries generated by randomization of one codon, 190 colonies (approximately six-fold the

399 theoretical number of gene variants generated from an NNK codon, where $\mathrm{N}=\mathrm{A}, \mathrm{G}, \mathrm{C}, \mathrm{T}$ and $\mathrm{K}$

$400=\mathrm{G}, \mathrm{T}$ ) were picked randomly and screened. For libraries generated by randomization of a

401 combination of two codons, 570 green fluorescent colonies (approximately 50\% of the theoretical

402 number of gene variants) were picked and screened.

403 For each cultured variant, protein was extracted using $50 \mu \mathrm{L} 0.5 \%$ of $n$-octyl- $\beta$-D-

404 thioglucopyranoside in Tris buffered saline (TBS, pH 8) and then further diluted 1:4 in PBS buffer

405 ( $\mathrm{pH}$ 7.4). Photoconversion was performed in transparent microplates (Nunc-Thermo Fisher

406 Scientific; Mfr.No. 265302) under a $405 \mathrm{~nm}$ LED flood array (Loctite) with an intensity of 3.46

$407 \mathrm{~mW} / \mathrm{mm}^{5}$. Diluted samples $(100 \mu \mathrm{L})$, with and without photoconversion, were tested on 96-well 408 plates (Thermo Fisher Scientific) at 5 min after photoconversion. To each well was added $10 \mu \mathrm{L}$

409 luciferase substrate furimazine in PBS solution $(0.1 \mathrm{mg} / \mathrm{mL})$, and the luminescence was measured 410 immediately using a Cytation 5 plate reader (BioTek). The top $2 \%$ variants with bright 
411 bioluminescence and large intensity decrease (> 60\% decrease) were picked for sequencing and

412 used as templates for the next evolution. A total of 34 libraries with 46 targeted amino acids were

413 screened for the identification of the $\mathrm{PhoCl} 2 \mathrm{c}$ and $\mathrm{PhoCl} 2 \mathrm{f}$ variants.

414 Protein purification and in vitro characterization.

To purify protein for crystallization, the gene expressing $\mathrm{PhoCl}$ with a $6 \times \mathrm{His}$ tag at the $\mathrm{C}$ -

416 terminal in pET-28a vector was used to transform E. coli strain BL21 (DE3) pLysS (Promega). A

417 single colony was picked to inoculate a $5 \mathrm{~mL}$ starting culture that was allowed to grow overnight

418 at $37{ }^{\circ} \mathrm{C}$ with $225 \mathrm{rpm}$ before being diluted into large cultures (1 liter for PhoCl green state and

419 red state; 10 liter for PhoCl empty barrel) of LB media supplemented with $100 \mu \mathrm{g} / \mathrm{mL}$ ampicillin.

420 The cultures were allowed to grow at $37^{\circ} \mathrm{C}$ to an $\mathrm{OD}_{600}$ of 0.5 , and then induced with IPTG $(0.1$

$421 \mathrm{mM}$ ) and grown overnight at $28^{\circ} \mathrm{C}$. The $E$. coli cells were harvested by centrifuge and lysed by

422 sonication. Protein was purified by Ni-NTA chromatography (G-Biosciences). For PhoCl empty

423 barrel purification, the intact $6 \times$ His tag fused $\mathrm{PhoCl}$ protein was captured by Ni-NTA agarose and

424 was photoconverted on the column with $10 \times 15 \mathrm{~s}$ illumination (LED array, $3.46 \mathrm{~mW} / \mathrm{mm}^{2}$ ), and

425 the eluent containing the PhoCl empty barrel was collected.

426

To prepare the proteins for in vitro characterizations of $\mathrm{PhoCl}$ variants, the E. coli strain

427 DH10B were transformed with the $\mathrm{pBAD} / \mathrm{HisB}$ vector encoding the gene of interest. Cultures were

428 induced with L-arabinose $(0.2 \%)$ and allowed to grow $24 \mathrm{~h}$ at $30{ }^{\circ} \mathrm{C}$. Protein were purified by $\mathrm{Ni}$ -

429 NTA chromatography. Molar extinction coefficients $(\varepsilon)$ at $488 \mathrm{~nm}$ were determined by the alkali

430 denaturation method ${ }^{39}$ and then used as reference $\varepsilon$ for the $405 \mathrm{~nm}$. Quantum yields for PhoCl

431 variants were measured using purified EGFP as the reference standard ${ }^{40}$. Photoconversion was

432 performed with the $405 \mathrm{~nm}$ LED flood array. The absorbance spectra were acquired with a DU-

$433800 \mathrm{UV}$-vis spectrophotometer (Beckman). The fluorescence spectra were acquired with a Safire2 
434 plate reader (Tecan) and bioluminescence spectrums were acquired with a Cytation 5 plate reader

435 (BioTek). The gel filtration was performed with a HiPrep 16/60 Sephacryl S-100 column on an

436 AKTA chromatography system (GE Healthcare), and the size of the proteins in each eluted fraction

437 were verified by SDS-PAGE.

\section{8}

439

440

441

442

443

444

445

446

447

448

449

450

451

\section{2}

453

454

455

456

\section{Protein crystallization and $\mathrm{X}$-ray data collection.}

The green state of PhoCl1 and PhoCl1 empty barrel recombinant proteins were further purified with size exclusion chromatography using SD200 column (GE Healthcare) and buffer exchanged to $20 \mathrm{mM}$ Tris $\mathrm{pH} 7.4,150 \mathrm{mM} \mathrm{NaCl}$. Initial crystallization trials were carried out with 384-well plate via sitting drop vapor diffusion against commercially available sparse matrix screens (Hampton Research and Molecular Dimensions) at room temperature. The green state of PhoCl1 was crystallized in $0.1 \mathrm{M}$ MIB buffer $\mathrm{pH}$ 6.0, 25\% w/v PEG 1500. Crystals of the red state of PhoCl1 was generated from the PhoCl1 green crystals through photoconversion with the violet light (15 s illumination with $405 \mathrm{~nm}$ LED array, $3.46 \mathrm{~mW} / \mathrm{mm}^{2}$ ). The PhoCl1 empty barrel crystals were observed in $0.056 \mathrm{M} \mathrm{NaH}_{2} \mathrm{PO}_{4} \cdot \mathrm{H} 2 \mathrm{O}, 1.344 \mathrm{M} \mathrm{K}_{2} \mathrm{HPO}_{4}, \mathrm{pH}$ 8.2. All the crystals were cryoprotected with the reservoir condition in supplement with $15-20 \%$ glycerol and flash frozen in liquid nitrogen. X-ray diffraction dataset were collected at Canadian Light Source CMCF-ID beamline. All the datasets were processed and scaled with XDS package ${ }^{41}$; the data collection details and statistics are summarized in Supplementary Table 1.

\section{Structure determination and refinement.}

The structure of the green state of PhoCl1 was solved by molecular replacement using mTFP1 (PDB ID: 2HQK) as search model ${ }^{42,43}$. The red state of PhoCl1 and PhoCl1 empty barrel structure determination were carried out using the solved the green state of PhoCl1 as template.

The manual model building and refinement were performed with $\operatorname{Coot}^{44}$ and PHENIX ${ }^{45}$ refine. 
457 The CR8 and IEY were used as the chromophore ligands for the green state and the red state of

458 PhoCl1 respectively. In the PhoCl1 red crystal, the chromophore turned out to be partially

459 converted, therefore two of the six monomers in the asymmetric unit with high occupancy for IEY

460 were modeled in red form, and the other four monomers were built in green form.

461 The PhoCl1 green form was solved to $2.10 \AA$ with a $R_{W o r k}$ factor of 0.1824 and $R_{\text {Free }}$ factor

462 of 0.2162 in $\mathrm{P} 2{ }_{1} 2{ }_{1}{ }_{1}$ spacegroup. The Photoconversion of the PhoCl1 green crystal induced the

463 packing of the crystal and the PhoCl1 red model was refined to $2.30 \AA$ with a $R_{W o r k}$ factor of 0.2186

464 and $R_{\text {Free }}$ factor of 0.2638 in P1 spacegroup. The PhoCl1 empty barrel was determined to $2.82 \AA$

465 in $\mathrm{P} 2{ }_{1}$ spacegroup with anisotropical diffraction; the dataset was processed with the Diffraction

466 Anisotropy Server $^{46}$ and refined with a $R_{W o r k}$ factor of 0.2587 and $R_{\text {Free }}$ factor of 0.3029.

467 All the structures showed favorable stereochemistry and exhibited good distribution of 468 dihedral angles on a Ramachandran plot. Detailed refinement statistics are summarized in

469 Supplementary Table 1. Structure figures were generated using the molecular visualization 470 PyMOL package (version 1.8) ${ }^{47}$ and UCSF Chimera (production version 1.13.1) ${ }^{48}$. Schematic

471 diagram of the dissociable peptide in the PhoCl1 red state was generated using LIGPLOT (version $472 \quad 4.5 .3)^{49}$

\section{Molecular dynamic (MD) simulation.}

474 To prepare the starting structure for MD, the red state chromophore in the protonated state, 475 designated as "RCP", was generated in Avogadro (version 1.2.0) ${ }^{50}$, and parameterized using

476 R.E.D. Server Development 2.0 (Ref. 51). The circular cp linker (residues 147-172), which was

477 not resolved in the crystal structure of the photo-converted PhoCl1, was modelled in Rosetta

478 (version 2018.33.60351) using the kinematic closure (KIC) with fragment protocol ${ }^{52}$. Briefly,

47910,000 models were generated and the structure with the largest negative score was selected to 
480 represent the starting conformation of the cp linker. The experimental structure of the PhoCl1 red

481 state structure, edited to include the parameterized RCP chromophore and the cp linker, was

482 subsequently solvated in LEaP (AmberTools19) with explicit water (TIP3P) and 1 sodium

483 counterion in an octagon periodic boundary condition with each side no closer than $30 \AA$ away

484 from the protein.

All MD simulations are performed with Amber18 with GPU parallelization on Cedar cluster, Compute Canada. The system was minimized, heated to $303.15 \mathrm{~K}$ and equilibrated with

487 decreasing restraints for the protein. An unconstrained production simulation (cMD) was carried 488 out at $303.15 \mathrm{~K}$ for $210 \mathrm{~ns}$ under isothermal-isobaric (NPT) ensemble. The restart file at the end 489 of the cMD served as the initial coordinate of the adaptive steered MD (ASMD). The distance 490 between the centres-of-mass (COMs) for the barrel (residues 1-231) and the C-terminal peptide 491 (residues 232-242) were subjected to a harmonic constraint (rk2=18) increasing by $40 \AA$ over 40 492 ns, without specifying the direction of dissociation. Such process was divided into 40 stages, where 493 the distance between the COMs increased by $1 \AA$ over $1 \mathrm{~ns}$ in each stage with 100 replications. 494 The average of potential of mean force (PMF) was calculated by Jarzynski Equality ${ }^{53}$ after all 495 replicates had completed. The replicate closest to the average was chosen to represent the trajectory 496 of that stage whose restart file served as the initial coordinate of the next stage. To investigate 497 whether the conformation of the cp linker affects the dissociation pathway, the same ASMD 498 simulation was repeated twice restarting from $150 \mathrm{~ns}$ and $200 \mathrm{~ns}$ of the cMD, respectively. The 499 trajectories of the chosen replicate from each stage were concatenated and aligned with the 500 program CPPTRAJ (AmberTools 19) ${ }^{54}$. Free energy along the dissociation process was calculated 501 using molecular mechanics/Poisson-Boltzmann surface area (MM/PBSA) method quasi-harmonic 
502

503

504

505

506

507

508

509

510

511

512

513

514 at the ends of $\mathrm{PhoCl}$, was made using overlap extension PCR to connect three gene fragments (i.e.,

515 NBid, PhoCl, and CBid). The assembled gene (with 5' NheI and 3' XhoI restriction sites) was

516 digested and inserted between the NheI and XhoI sites of pcDNA 3.1(+), thus yielding the PhoCl

517 inserted Bid expression vector.

518

519 kinase kinase (MAPKK) ${ }^{32}$ and caspase-3 substrate (DEVD) was appended by PCR to the 5' end

520 of the gene encoding mCardinal, and DNA encoding the $3 \times$ NLS of SV40 (Ref. 57) was appended

521 to the 3' end of fragment. The gene encoding NES-DEVD-mCardinal-NLS was then inserted

522 between NheI and XhoI sites of pcDNA 3.1(+), thus yielding the caspase-3 reporter expression

523 vector. 
For expression of NES-PhoCl-mCherry, HeLa (ATCC CCL-2) cells at 70\% confluency in

525

526

527

528

529

530

531

532

533

534

535

536

537

538

539

540

541

542

543

544

545

546

35-mm cell culture dishes (Corning) were transfected with $2 \mu \mathrm{g}$ plasmid DNA and $4 \mu \mathrm{L}$ Turbofect

(Thermo Fisher Scientific) according to the manufacturer's protocol. Imaging was performed at

$24 \mathrm{~h}$ after transfection. For expression of $\mathrm{PhoCl}$ inserted Bid and caspase-3 reporter, HeLa cells

were co-transfected with $1.75 \mu \mathrm{g}$ NES-DEVD-mCardinal-NLS expression vectors and $0.25 \mu \mathrm{g}$

$\mathrm{PhoCl}$ inserted Bid expression vectors mixed with $4 \mu \mathrm{L}$ Turbofect. For expression of $\mathrm{PhoCl}$ inserted Bid for the DEAD viability assay, $1.75 \mu \mathrm{g}$ pcDNA 3.1(+) empty vector and $0.25 \mu \mathrm{g}$

PhoCl-Bid were used per transfection. The transfection was performed in $2 \mathrm{~mL}$ of serum-free DMEM for $2 \mathrm{~h}$, after which the medium containing the transfection reagent and DNA mixture was replaced with $2 \mathrm{~mL}$ of complete medium supplemented with $10 \%$ fetal bovine serum (FBS; SigmaAldrich). Transfected cells were cultured $16 \mathrm{~h}$ before experiments.

\section{Cell photoconversion and imaging conditions.}

Before imaging, the medium was changed to HEPES-buffered Hanks Balanced Salt Solution (HHBSS; 20 mM HEPES). Wide-field fluorescence imaging was performed using a Nikon Eclipse Ti-E epifluorescence microscope equipped with a 75-W Nikon xenon lamp and a Photometrics QuantEM 512SC camera. The NIS-Elements AR package software was used for automatic instrument control and image acquisition. Cells were imaged with a $20 \times$ air objective lens (NA 0.75; Nikon) using the following filter sets: PhoCl green (490/15 nm excitation: ex; 510 nm dclp dichroic and 525/50 nm emission: em), mCherry and DEAD stain (543/10 nm ex, $565 \mathrm{~nm}$ dclp dichroic and 620/60 nm em), photoconversion (395/40 nm ex and $425 \mathrm{~nm}$ dclp dichroic), and mCardinal (605/50 $\mathrm{nm}$ ex, $635 \mathrm{~nm}$ dclp dichroic and 670/50 $\mathrm{nm}$ em).

For imaging of NES-PhoCl-mCherry protein translocation, the images were acquired in both the PhoCl green and mCherry channel every $15 \mathrm{~s}$ for $2 \mathrm{~min}$ before photoconversion. 
547 Photoconversion was performed with $10 \mathrm{~s}$ pulse illumination $\left(2 \mathrm{~mW} / \mathrm{mm}^{2}\right)$ every $15 \mathrm{~s}$ for $6 \mathrm{~min}$

548 and each pulse of photoconversion light was followed by acquisition of $\mathrm{PhoCl}$ and mCherry

549 fluorescence images. Imaging acquisition of green and red channels continued every $15 \mathrm{~s}$ for

550 another $15 \mathrm{~min}$ after the photoconversion. Protein translocation was analyzed as the ratio of

551 intensity of mCherry in the cytoplasm to the nucleus. Photoconversion efficiency was determined

552 by the loss of green fluorescence intensity in the cytoplasm.

553

For imaging the caspase-3 activity triggered by photo-induced cell death with $\mathrm{PhoCl}$

554 inserted Bid, images were acquired in both the PhoCl green and mCardinal channel every $15 \mathrm{~s}$ for

5552 min before photoconversion. Photoconversion was performed with $10 \mathrm{~s}$ pulse illumination $(2$

$556 \mathrm{~mW} / \mathrm{mm}^{2}$ ) every $15 \mathrm{~s}$ for $6 \mathrm{~min}$ and each pulse conversion light was followed by acquisition of

$557 \mathrm{PhoCl}$ and mCardinal fluorescence images. Imaging acquisition of green and red channels

558 continued every $30 \mathrm{~s}$ for $2 \mathrm{~h}$ after the photoconversion. Caspase- 3 activation was determined by

559 the translocation of mCardinal from cytoplasm into nucleus.

560 For imaging of the DEAD stained HeLa cells with PhoCl inserted Bid expression,

561 photoconversion was performed under the $405 \mathrm{~nm}$ LED flood array with $15 \mathrm{~s}$ illumination.

562 Following photoconversion, the cells were incubated in HHBSS buffer (with or without Z-VAD-

563 FMK) at room temperature for $30 \mathrm{~min}$. The working concentration of Z-VAD-FMK (Sigma-

564 Aldrich) was $20 \mu \mathrm{M}$. For analysis the cell death, the DEAD Cytotoxicity kit for mammalian cells

565 (Invitrogen) was used to stain the dead cells with or without photoconversion in HHBSS buffer

566 (with or without Z-VAD-FMK) for $30 \mathrm{~min}$ at room temperature according to the manufacturer's

567 protocol. Images were acquired in white light, green fluorescence and red fluorescence channels.

568 The extent of cell death was determined by the ratio of DEAD reagent stained cells to the green

569 fluorescent transfected cells. 


\section{Statistical analysis.}

571 All data are expressed as mean \pm SEM. Sample sizes $(n)$, significant differences $(P)$ and confidence

572 of curve fitting ( $R$-squared) are listed for each experiment. Results are reported as $P=P$ value, and

$573 t($ degrees of freedom $(\mathrm{df}))=t$ value. For all statistics, NS $=P \geq 0.05, *=P<0.05, * *=P<0.01$,

$574 * * *=P<0.001$ and $* * * *=P<0.0001$. No samples were excluded from analysis and all

575 experiments were reproducible. No randomization or blinding was used. The analyses were 576 performed using GraphPad Prism for Mac (version 8.0.0), GraphPad Software, San Diego,

577 California USA, www.graphpad.com.

\section{Data availability}

579 Expression vectors for the following gene products and their gene sequences are available via 580 Addgene and GenBank: pET-PhoCl1-6His, Addgene ID: 164033, and GenBank accession no. 581 MW296870; pBad-LgBiT-PhoCl1-SmBiT-MBP, Addgene ID: 164034, and GenBank accession 582 no. MW307773; pBad-PhoCl2c, Addgene ID: 164035, and GenBank accession no. MW296871; 583 pBad-PhoCl2f, Addgene ID: 164036, and GenBank accession no. MW307774; pcDNA-NES584 PhoCl2c-mCherry, Addgene ID: 164037, and GenBank accession no. MW307775; pcDNA-NES585 PhoCl2f-mCherry, Addgene ID: 164048, and GenBank accession no. MW307776; pcDNA-NBid586 mMaple-CBid, Addgene ID: 164049, and GenBank accession no. MW307777; pcDNA-NBid-

587 PhoCl1-CBid, Addgene ID: 164050, and GenBank accession no. MW307778; pcDNA-NBid588 PhoCl2c-CBid, Addgene ID: 164051, and GenBank accession no. MW307779; pcDNA-NES589 DEVD-mCardinal-NLS, Addgene ID: 164052, and GenBank accession no. MW307780. Other 590 expression vectors that support the findings in this study are available from the corresponding 591 author on request. The primers used in this study and nucleotide sequences of all constructs and 592 the are available in Supplementary Table 7,8. The coordinates of the PhoCl1 green, red and 
593 empty barrel structures are deposited in the Protein Data Bank under the codes of 7DMX, 7DNA

594 and 7DNB, respectively.

\section{Acknowledgements}

596 This work was supported by grants from the Canadian Institutes of Health Research (FS- 154310

597 to REC) and the Natural Sciences and Engineering Research Council of Canada (RGPIN 2018-

59804364 to REC and RGPIN-2016-06478 to MJL). Y. W. is supported by the Alberta Parkinson

599 Society Fellowship and National Natural Science Foundation of China (NO. 31870132 and NO.

600 82072237). S. Z. is supported by NSERC CREATE Advanced Protein Engineering Training,

601 Internships, Courses, and Exhibition (APRENTICE) program. Part of the research described in

602 this paper was performed using beamline CMCF-ID at the Canadian Light Source, a national

603 research facility of the University of Saskatchewan, which is supported by the Canada Foundation

604 for Innovation (CFI), the Natural Sciences and Engineering Research Council (NSERC), the

605 National Research Council (NRC), the Canadian Institutes of Health Research (CIHR), the

606 Government of Saskatchewan, and the University of Saskatchewan. This research was enabled in

607 part by support provided by WestGrid (https://www.westgrid.ca/) and Compute Canada

608 (www.computecanada.ca). We thank the University of Alberta Molecular Biology Services Unit,

609 Gareth Lambkin, and Landon Zarowny for technical assistance. The furimazine luciferase

610 substrate was synthesized by the Canadian Glycomics Network.

\section{Author Contributions}

612 X. L. developed PhoCl2 variants, assembled all constructs, performed directed evolution,

613 performed protein characterization, performed live cell imaging experiments, analyzed data,

614 prepared figure, and wrote the manuscript. Y. W. performed the protein crystallization, solved the 
615 structures, and wrote the manuscript. S. Z. performed the molecular dynamic simulation, prepared 616 figures, and wrote the manuscript. W. Z. conceived the idea of light-induced cell death experiment, 617 performed the proof-of-concept tests, and prepared the figure. Y. C. performed the molecular 618 dynamics simulation and the analysis. Y. S., M. J. L., and R. E. C. supervised research and edited 619 the manuscript. 
a

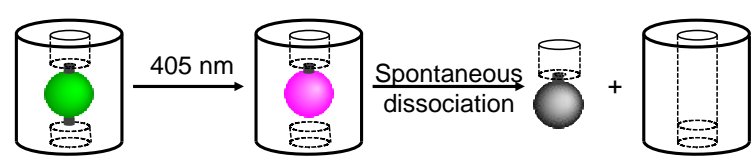

b

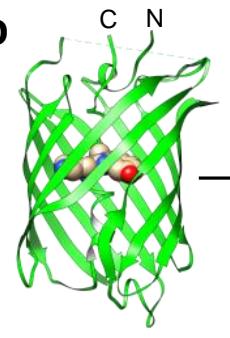

C

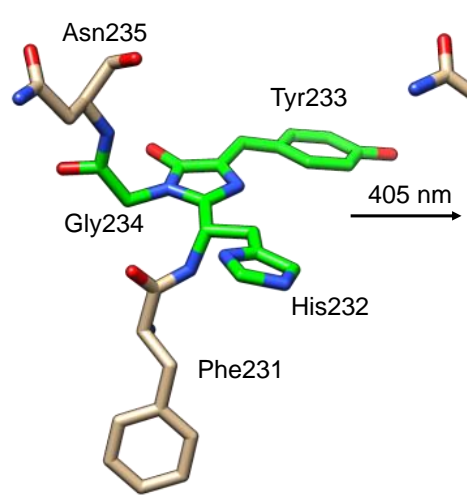

C $\mathrm{N}$

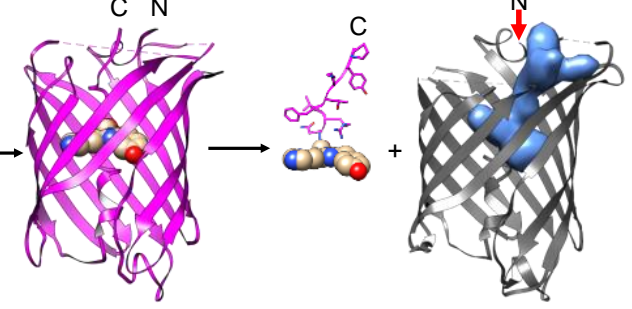

d

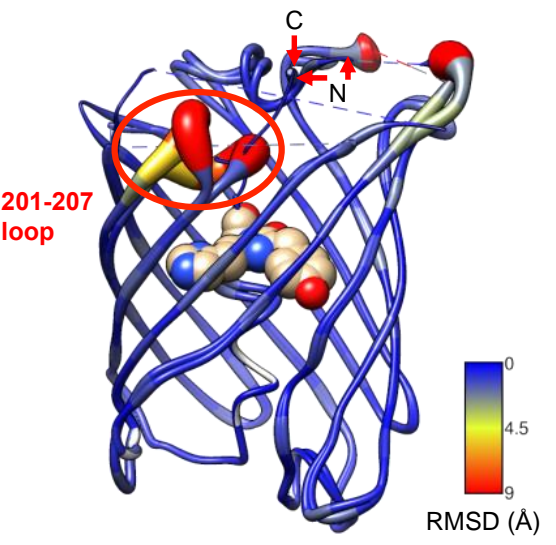

621

e

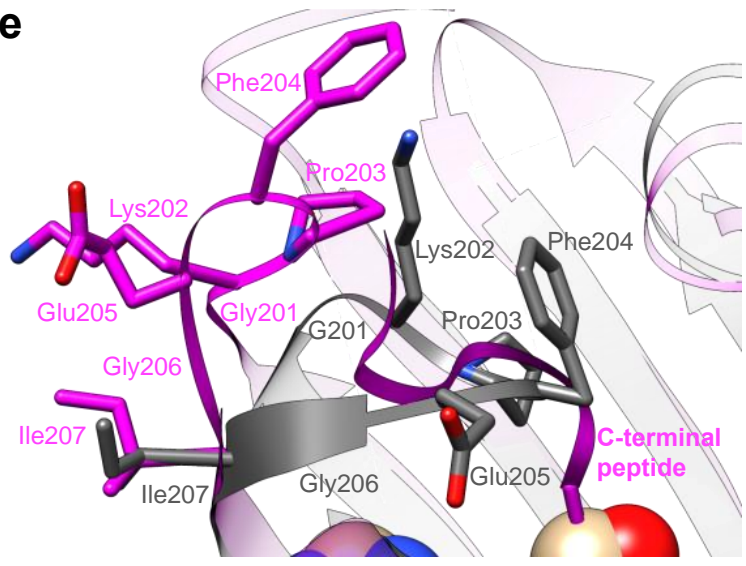

622 Figure 1. Overview of PhoCl1 structure and function. (a) Schematic of PhoCl photoconversion

623 and dissociation. (b) Representation of PhoCl1 structures. Green state of PhoCl1: green structure

624 (PDB ID: 7DMX), the red state: magenta structure (PDB ID: 7DNA), and the cleaved PhoCl1

625 empty barrel: grey structure (PDB ID: 7DNB). Water-filled cavity is represented as a blue space-

626 filling volume. Dissociated peptide fragment is shown as sticks for the peptide portion and spheres

627 for the chromophore portion. (c) The experimentally-determined structure of the PhoCl1

628 chromophore in the green and red states. (d) Structure alignment of the PhoCl1 red state and empty

629 barrel colored by RMSD values. The RMSD values are indicated by scale bar and coil thickness

630 (thicker coil indicates higher RMSD). (e) Zoomed-in representation of the 201-207 loop in the

631 aligned structures of the red state (magenta) and empty barrel (grey). 


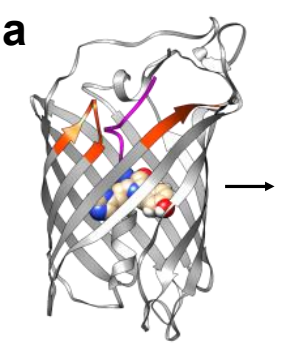

Initial

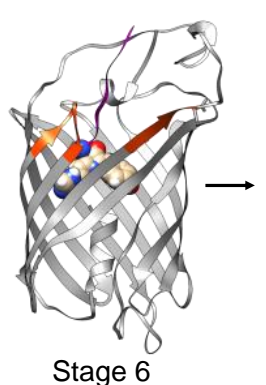

Stage 6

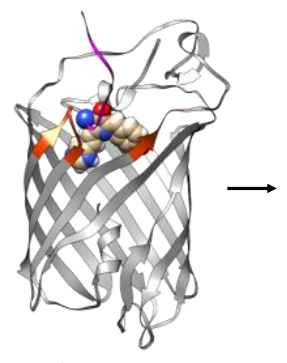

Stage 10

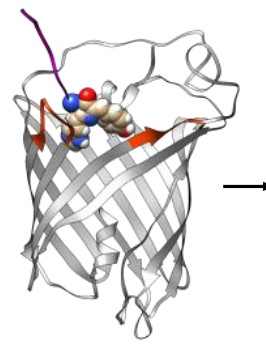

Stage 17

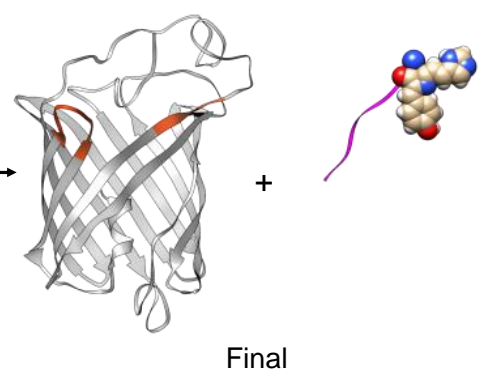

b
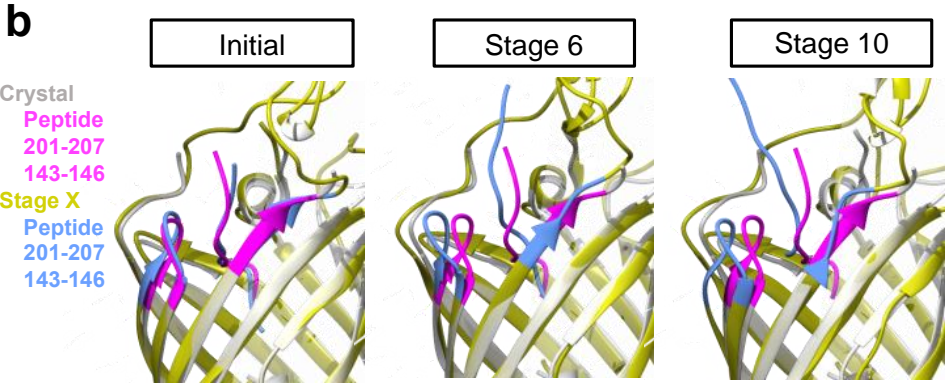

C
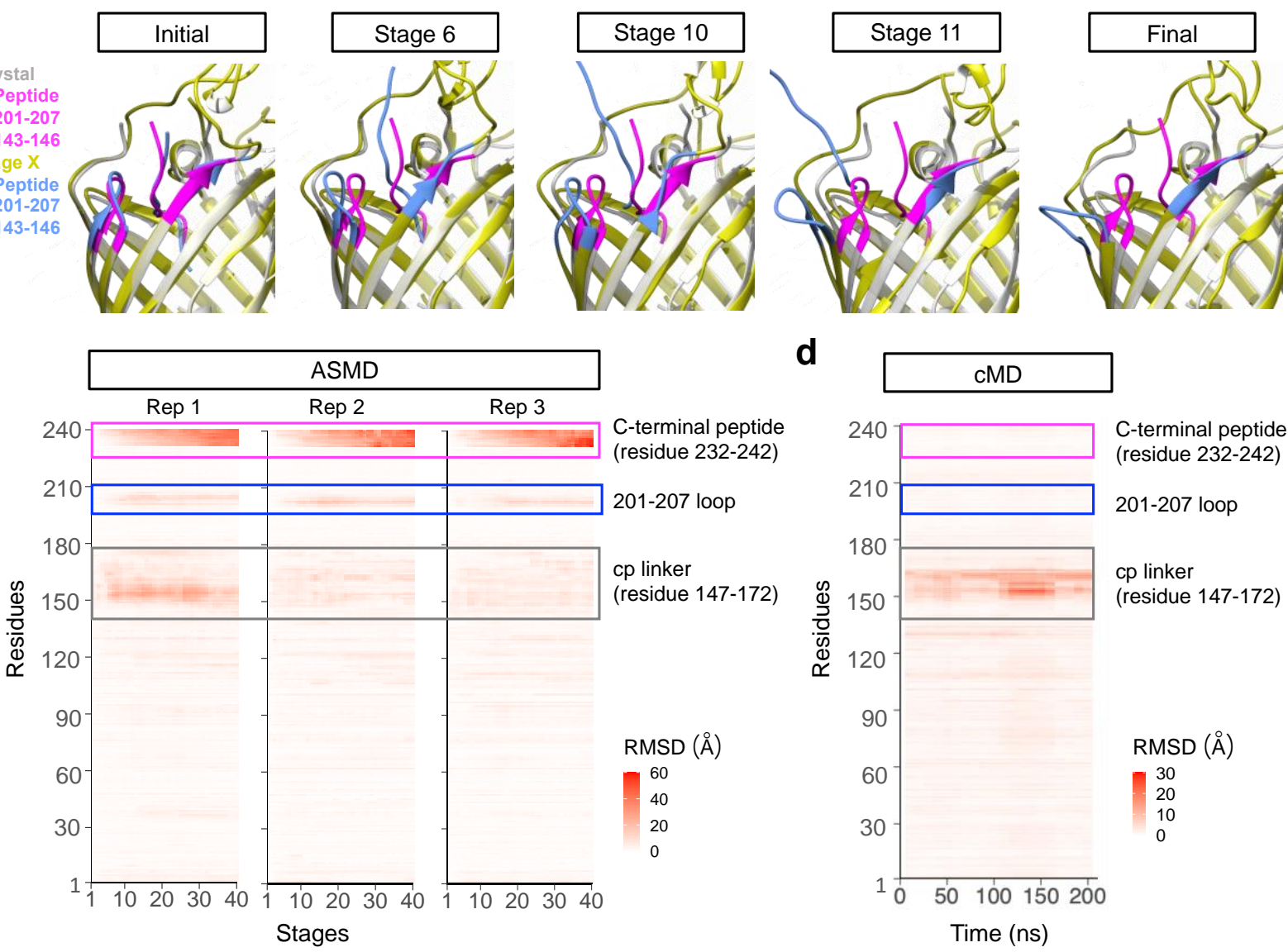

d
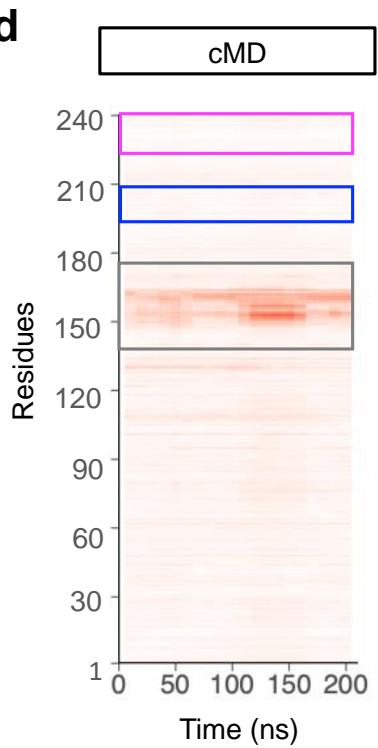

C-terminal peptide (residue 232-242) 201-207 loop

cp linker (residue 147-172)

$\operatorname{RMSD}(\AA)$

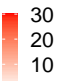

20
10
0

Figure 2. Molecular dynamic simulation on PhoCl1 dissociation process. (a) Representation

634 of the simulated dissociation process. Photo-converted PhoCl1 is shown in grey, dissociated

635 peptide fragment is shown as magenta ribbon for the peptide portion and spheres for the 636 chromophore. Residues within and near the 201-207 loop are highlighted in orange. (b) Structure

637 alignment of the crystal structure of the PhoCl1 red state and the simulated structure from different 638 stages of ASMD replication 3 (Rep 3). Crystal structure of the PhoCl1 red state is shown in silver, 
639 with residues within and near the 201-207 loop highlighted in magenta. Simulated structures from

640 different stages are shown in yellow, with residues within and near the 201-207 loop highlighted

641 in blue. (c) RMSD heatmaps of 3 ASMD replications over stages with the initial stage as reference.

642 The RMSD values were colored according to the scale bar. The C-terminal peptide is enclosed in

643 a magenta box, the 201-207 loop is enclosed in a blue box and the cp linker is enclosed in a grey

644 box. Rep, replication. (d) RMSD heatmaps of cMD over stages with the initial stage as reference.

645 The RMSD values were colored according to the scale bar. 
a

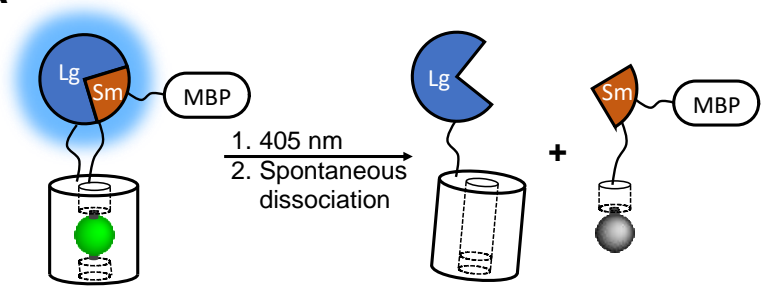

b

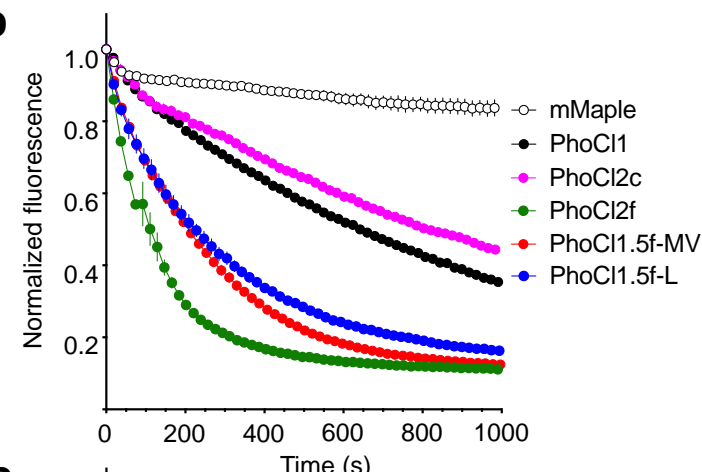

C

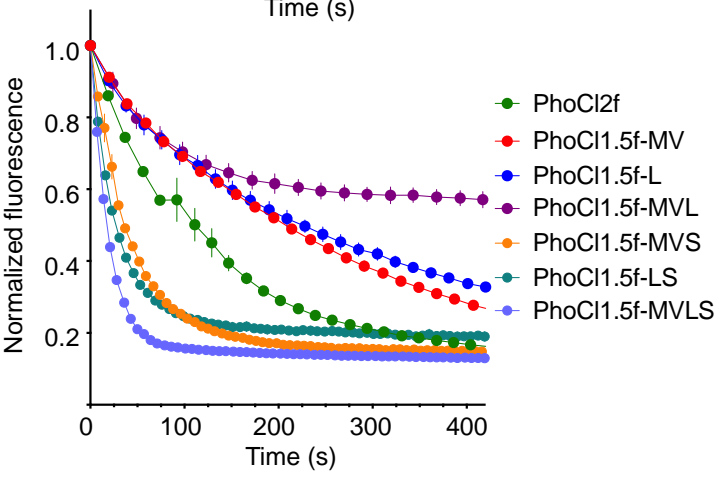

d

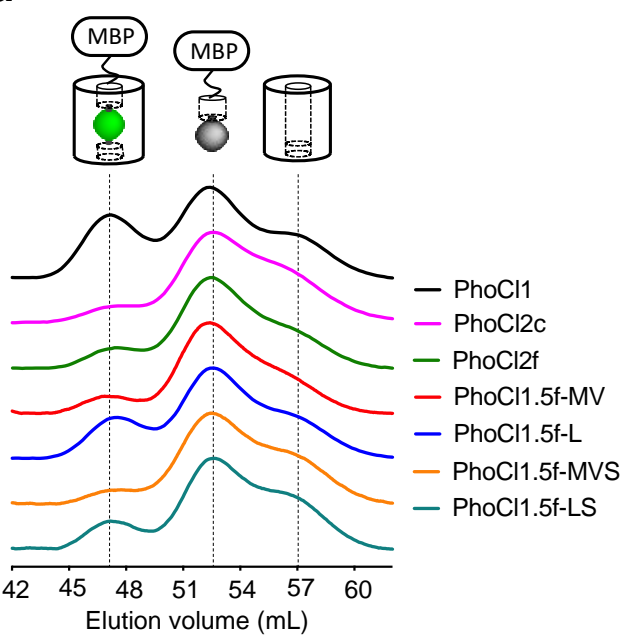

e

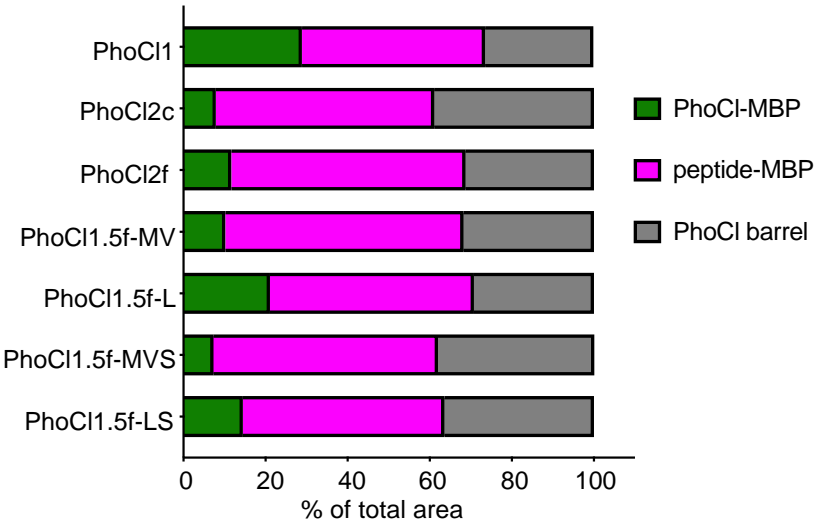

Figure 3. Library screening assay and characterization of PhoCl2 variants. (a) Schematic of fluorescence after photoconversion. The red fluorescence was monitored immediately following

$65115 \mathrm{~s}$ illumination with $405 \mathrm{~nm}$ light (LED array, $3.46 \mathrm{~mW} / \mathrm{mm} 2)$. Values are means $\pm \mathrm{SEM}(n=3$

652 independent experiments). One phase decay fit was used to determine the dissociation half time,

$653 t_{1 / 2} . R$-squared values for fits range from 0.9830 to 0.9975. (c) Dissociation kinetics of PhoCl2f

654 and PhoCl1.5f variants with the combinations of key mutations. (d) Gel-filtration chromatography

655 (GFC) analysis of maltose binding protein (MBP) fused $\mathrm{PhoCl}$ variants. All proteins were tested

656 at the same concentration $(2 \mathrm{mg} / \mathrm{mL})$ and illumination conditions (15 s LED array illumination, 
$6573.46 \mathrm{~mW} / \mathrm{mm}^{2}$ ). Partial photoconverted sample was loaded on the HiPrep 16/60 Sephacry S-100

658 column immediately after illumination. The intact PhoCl-MBP fusion (75 kDa) was eluted first

659 from the column at a volume of $\sim 47 \mathrm{~mL}$. This was followed by the cleaved peptide-MBP fusion

$660(45 \mathrm{kDa})$ at $\sim 52 \mathrm{~mL}$ and then the cleaved empty barrel $(30 \mathrm{kDa})$ at a volume of $\sim 57 \mathrm{~mL}$. The

661 identity of the fragments associated with each peak in the eluent were confirmed through SDS-

662 PAGE analysis (in Supplementary Fig. 8c). (e) Summary of peak area ratios from the GFC

663 analysis. Baseline correction, multi-peak fitting, and peak area integration were performed using

664 the OriginLab software. A Gaussian fit model was used in peak fitting with three fixed widths

665 curves corresponding to the three elution peaks. $R$-squared values for peak fits range from 0.9974

666 to 0.9993. 
a

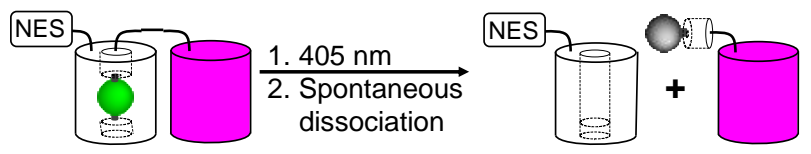

b
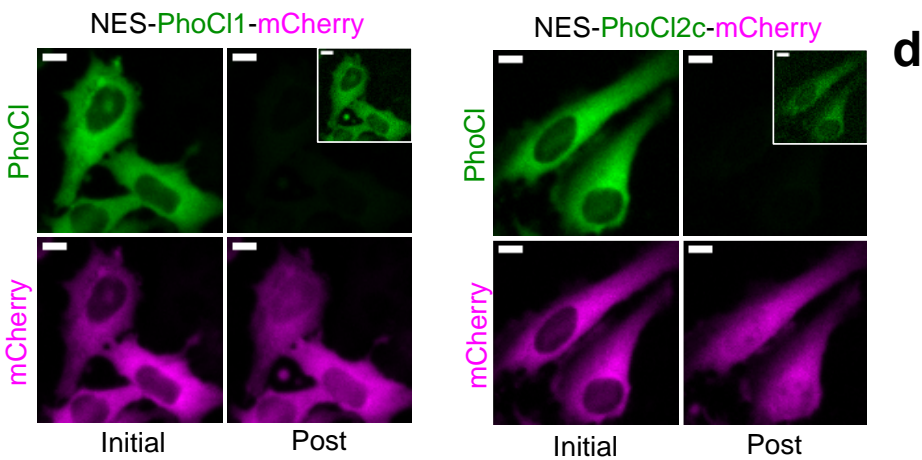

669

C
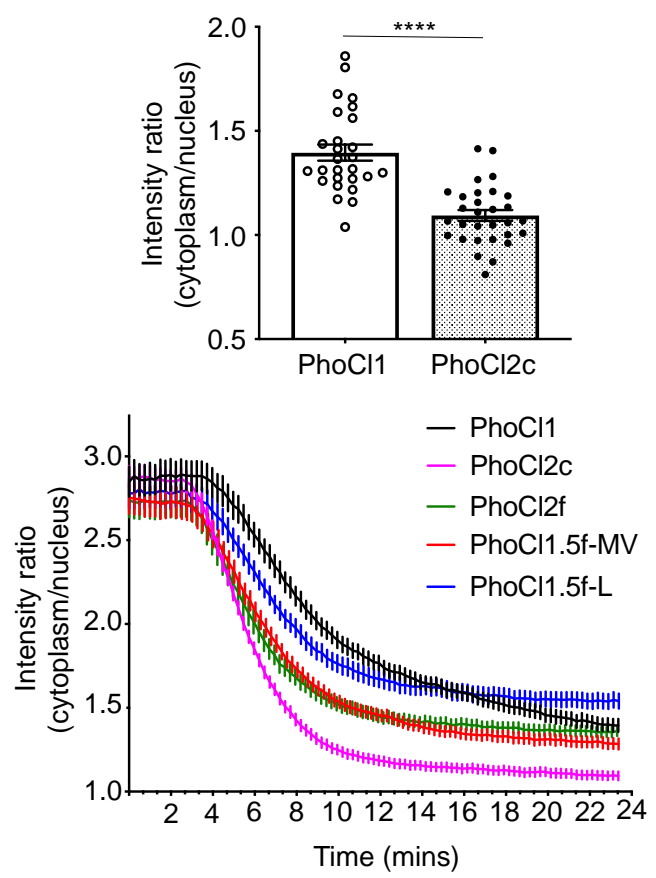

670 Figure 4. Optogenetic manipulation of protein translocation in HeLa cells. (a) Schematic of

671 the NES-PhoCl-mCherry photocleavage. (b) Representative images of HeLa cells expressing

672 NES-PhoCl-mCherry before and after $(15 \mathrm{~min})$ photoconversion. Conversion was performed with

$67310 \mathrm{~s}$ violet light pulses $\left(395 / 40 \mathrm{~nm}, 2 \mathrm{~mW} / \mathrm{mm}^{2}\right)$ every $15 \mathrm{~s}$ for 6 mins. Inset is the same image

674 with $10 \times$ increased contrast. Scale bar, $10 \mu \mathrm{m}$. (c) Red fluorescence intensity ratios of cytoplasm

675 to nucleus at 15 min after photoconversion. Ratios were calculated for single cells. Values are

676 means $\pm \operatorname{SEM}(n=27$ cells of PhoCl1, and $n=30$ cells of PhoCl2c). $* * * * P<0.0001$ by unpaired

677 two-tailed $t$ test $(t(55)=6.606)$. (d) Red fluorescence intensity localization ratios of cytoplasm to

678 nucleus versus time. Values are means \pm SEM $(n=27$ cells of PhoCl1 and PhoCl1.5f-L, and $n=$ 67930 cells of the other variants). 
a

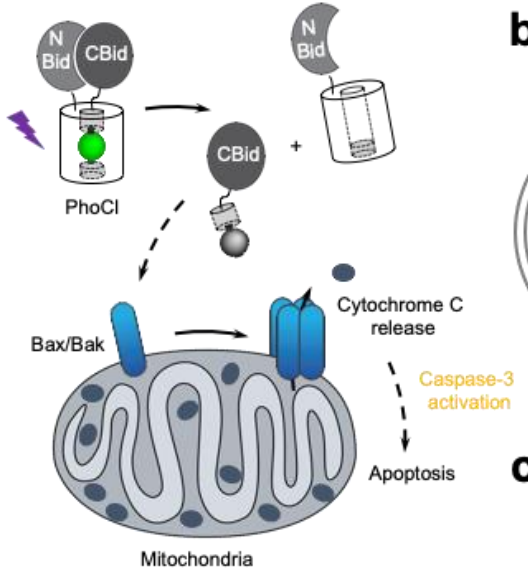

d

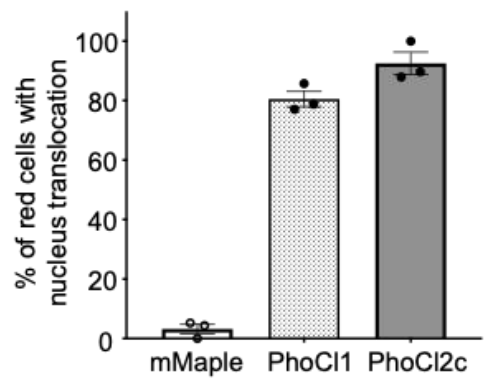

e

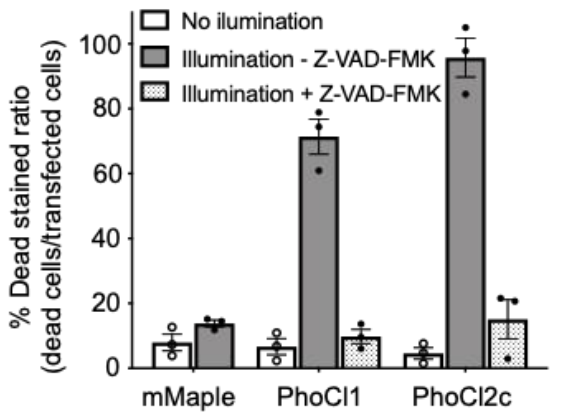

b

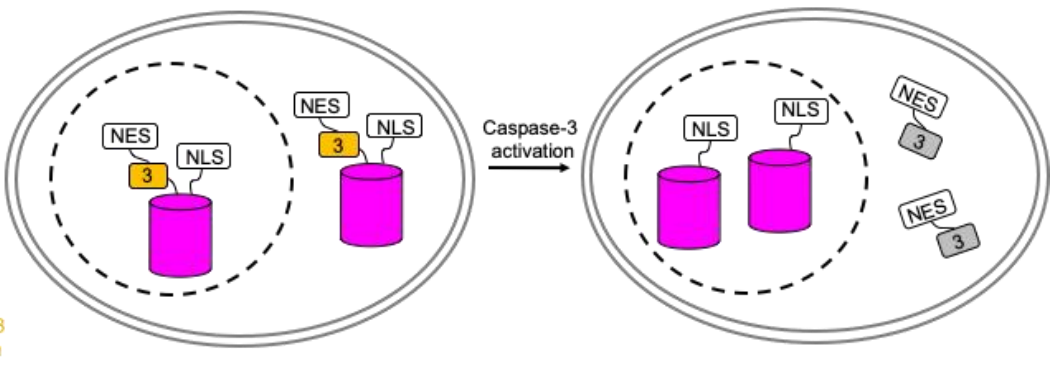

c

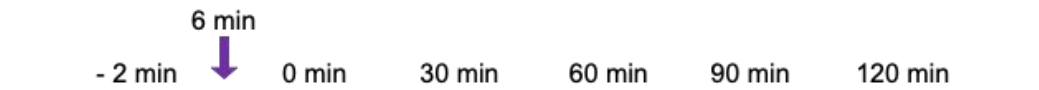

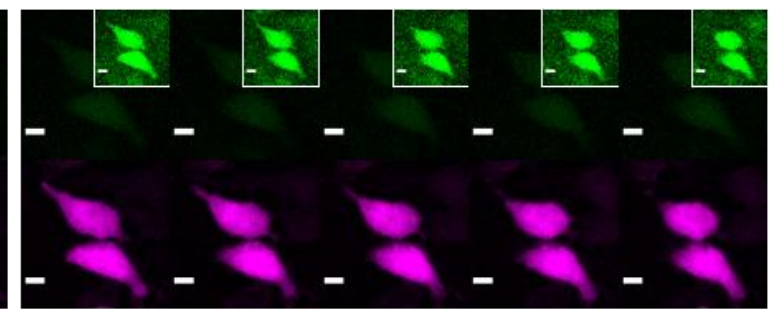
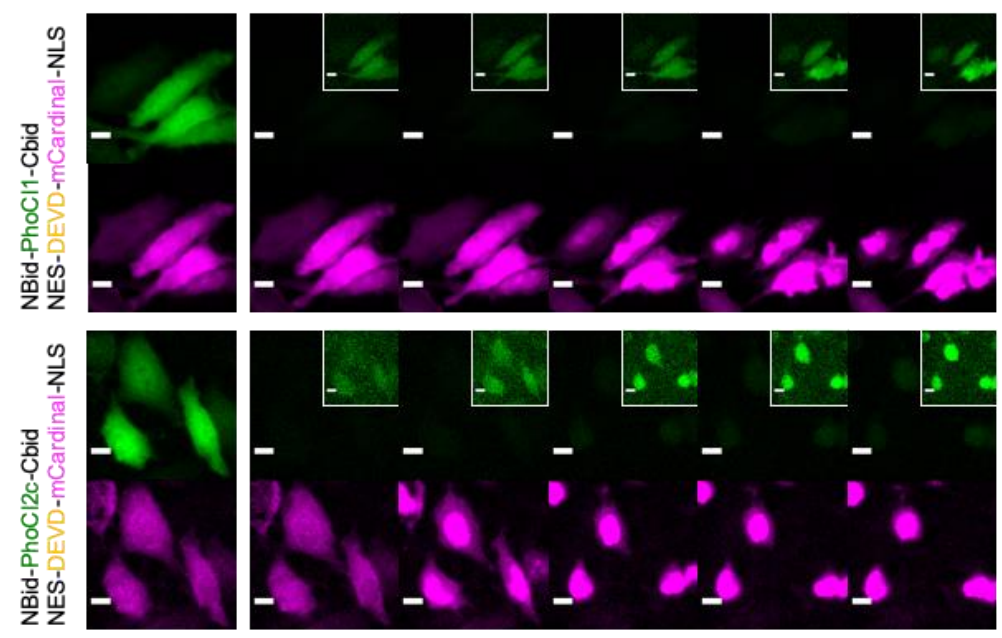

Figure 5. Optogenetic manipulation of cell apoptosis via PhoCl. (a) Schematics of optogenetic

683 activation of apoptosis with a PhoCl-Bid construct. (b) Schematics of apoptosis reporter: NES-

684 DEVD-mCardinal-NLS used in this experiment. (c) Transient transfected HeLa cells co685 expressing NBid-mMaple-CBid or NBid-PhoCl-CBid with caspase-3 reporter. Cells were 686 illuminated with $10 \mathrm{~s}$ violet light pulses $\left(395 / 40 \mathrm{~nm}, 2 \mathrm{~mW} / \mathrm{mm}^{2}\right)$ every $15 \mathrm{~s}$ for 6 mins. Inset is 687 the same image with $10 \times$ increased contrast. Scale bar, $10 \mu \mathrm{m}$. (d) Summary data of caspase-3 688 reporter translocation for cells expressing constructs described in c. Values are means $\pm \operatorname{SEM}(n=$ 
6893 cell cultures of each variant. A total of 87 cells for mMaple, 89 cells for PhoCl1 and 113 cells

690 for PhoCl2c). (e) Summary data of cell viability assay. Photoconversion was performed with $30 \mathrm{~s}$

691 pulse light (LED array, $3.46 \mathrm{~mW} / \mathrm{mm}^{2}$ ). Ratios are calculated by determining the ratio of DEAD

692 stained cells to green transfected cells. Values are means $\pm \operatorname{SEM}(n=3$ cell cultures of each variant).

693 A total of 321 cells for mMaple no illumination, 301 cells for mMaple illumination - Z-VAD-

694 FMK, 316 cells for PhoCl1 no illumination, 328 cells for PhoCl1 illumination - Z-VAD-FMK,

695326 cells for PhoCl1 illumination + Z-VAD-FMK, 325 cells for PhoCl2c no illumination, 337 cells

696 for PhoCl2c illumination - Z-VAD-FMK and 318 cells for PhoCl2c illumination + Z-VAD-FMK. 


\section{References}

698 1. Tischer, D. \& Weiner, O. D. Illuminating cell signalling with optogenetic tools. Nat. Rev.

$699 \quad$ Mol. Cell Biol. 15, 551-558 (2014).

700 2. Liu, Q. \& Tucker, C. L. Engineering genetically-encoded tools for optogenetic control of 701 protein activity. Curr. Opin. Chem. Biol. 40, 17-23 (2017).

702 3. Johnson, H. E. \& Toettcher, J. E. Illuminating developmental biology with cellular $703 \quad$ optogenetics. Curr. Opin. Biotechnol. 52, 42-48 (2018).

704 4. Lu, X., Shen, Y. \& Campbell, R. E. Engineering Photosensory Modules of Non-Opsin$705 \quad$ Based Optogenetic Actuators. Int. J. Mol. Sci. 21, 6522 (2020).

706 5. Zhang, W. et al. Optogenetic control with a photocleavable protein, Phocl. Nat. Methods $707 \quad \mathbf{1 4}, 391-394(2017)$.

708 6. Nagel, G. et al. Channelrhodopsin-2, a directly light-gated cation-selective membrane 709 channel. Proc. Natl. Acad. Sci. U. S. A. 100, 13940-13945 (2003).

$7107 . \quad$ Gradinaru, V., Thompson, K. R. \& Deisseroth, K. eNpHR: A Natronomonas 711 halorhodopsin enhanced for optogenetic applications. Brain Cell Biol. 36, 129-139 $712 \quad$ (2008).

713 8. Gradinaru, V. et al. Molecular and Cellular Approaches for Diversifying and Extending $714 \quad$ Optogenetics. Cell 141, 154-165 (2010).

$7159 . \quad$ Airan, R. D., Thompson, K. R., Fenno, L. E., Bernstein, H. \& Deisseroth, K. Temporally 716 precise in vivo control of intracellular signalling. Nature 458, 1025-1029 (2009).

717 10. Niopek, D. et al. Engineering light-inducible nuclear localization signals for precise 718 spatiotemporal control of protein dynamics in living cells. Nat. Commun. 5, (2014).

719 11. Niopek, D., Wehler, P., Roensch, J., Eils, R. \& Di Ventura, B. Optogenetic control of 
nuclear protein export. Nat. Commun. 7, 1-9 (2016).

721 12. Wu, Y. I. et al. A genetically encoded photoactivatable Rac controls the motility of living

722 cells. Nature 461, 104-108 (2009).

723 13. Strickland, D. et al. TULIPs: Tunable, light-controlled interacting protein tags for cell 724 biology. Nat. Methods 9, 379-384 (2012).

725 14. Kawano, F., Suzuki, H., Furuya, A. \& Sato, M. Engineered pairs of distinct photoswitches for optogenetic control of cellular proteins. Nat. Commun. 6, (2015).

727 15. Kennedy, M. J. et al. Rapid blue-light-mediated induction of protein interactions in living cells. Nat. Methods 7, 973-975 (2010).

729 16. Levskaya, A., Weiner, O. D., Lim, W. A. \& Voigt, C. A. Spatiotemporal control of cell 730 signalling using a light-switchable protein interaction. Nature 461, 997-1001 (2009).

731 17. Kaberniuk, A., Shemetov, A. A. \& Verkhusha, V. V. A bacterial phytochrome-based optogenetic system controllable with near-infrared light. Nat. Methods 13, 591-597

$733 \quad(2016)$

734 18. Zhou, X. X., Chung, H. K., Lam, A. J. \& Lin, M. Z. Optical Control of Protein Activity by 735 Fluorescent Protein Domains. Science. 338, 810-814 (2012).

736 19. Mizuno, H. et al. Photo-induced peptide cleavage in the green-to-red conversion of a 737 fluorescent protein. Mol. Cell 12, 1051-1058 (2003).

738 20. Shadish, J. A., Strange, A. C. \& Deforest, C. A. Genetically Encoded Photocleavable 739 Linkers for Patterned Protein Release from Biomaterials. J. Am. Chem. Soc. 141, 15619$740 \quad 15625(2019)$.

741 21. Xiang, D. et al. Hydrogels With Tunable Mechanical Properties Based on Photocleavable 742 Proteins. Front. Chem. 8, 1-9 (2020). 
743 22. Endo, M., Iwawaki, T., Yoshimura, H. \& Ozawa, T. Photocleavable Cadherin Inhibits

744 Cell-to-Cell Mechanotransduction by Light. ACS Chem. Biol. 14, 2206-2214 (2019).

745 23. Reed, E. H., Schuster, B. S., Good, M. C. \& Hammer, D. A. SPLIT: Stable Protein

746 Coacervation Using a Light Induced Transition. ACS Synth. Biol. 9, 500-507 (2020).

747 24. Hall, M. P. et al. Engineered luciferase reporter from a deep sea shrimp utilizing a novel

748 imidazopyrazinone substrate. ACS Chem. Biol. 7, 1848-1857 (2012).

749 25. Dixon, A. S. et al. NanoLuc Complementation Reporter Optimized for Accurate

Measurement of Protein Interactions in Cells. ACS Chem. Biol. 11, 400-408 (2016).

751 26. Tsien, R. Y. The green fluorescent protein. Annu. Rev. Biochem. 67, 509-544 (1998).

752 27. Hori, Y. Crystal structure of the Aequorea victoria green fluorescent protein.

753 Tanpakushitsu Kakusan Koso. 52, 1768-1769 (2007).

754 28. Nienhaus, K., Nienhaus, G. U., Wiedenmann, J. \& Nar, H. Structural basis for photoinduced protein cleavage and green-to-red conversion of fluorescent protein EosFP. Proc.

757 29. Tsutsui, H. et al. The E1 Mechanism in Photo-Induced $\beta$-Elimination Reactions for 758 Green-to-Red Conversion of Fluorescent Proteins. Chem. Biol. 16, 1140-1147 (2009).

759 30. McEvoy, A. L. et al. mMaple: A Photoconvertible Fluorescent Protein for Use in Multiple $760 \quad$ Imaging Modalities. PLoS One 7, (2012).

761 31. Xu, Y., Piston, D. W. \& Johnson, C. H. A bioluminescence resonance energy transfer (BRET) system: Application to interacting circadian clock proteins. Proc. Natl. Acad. Sci. U. S. A. 96, 151-156 (1999).

764 32. Henderson, B. R. \& Eleftheriou, A. A comparison of the activity, sequence specificity, 
(2000).

767 33. Esposti, M. D. The roles of Bid. Apoptosis 7, 433-440 (2002).

768 34. Billen, L. P., Shamas-Din, A. \& Andrews, D. W. Bid: A Bax-like BH3 protein. Oncogene 27, S93-S104 (2008).

770 35. Schug, Z. T., Gonzalvez, F., Houtkooper, R. H., Vaz, F. M. \& Gottlieb, E. BID is cleaved 771 by caspase- 8 within a native complex on the mitochondrial membrane. Cell Death Differ.

772

$$
\text { 18, 538-548 (2011). }
$$

773 36. Slee, E. A. et al. Benzyloxycarbonyl-Val-Ala-Asp (OMe) fluoromethylketone (ZVAD.FMK) inhibits apoptosis by blocking the processing of CPP32. Biochem. J. 315, 2124 (1996).

776 37. Campbell, R. E. Fluorescent-Protein-Based Biosensors: Modulation of Energy Transfer as a Design Principle. Anal. Chem. 81, 5972-5979 (2009).

778 38. Wang, H. et al. LOVTRAP: An optogenetic system for photoinduced protein dissociation. 779 Nat. Methods 13, 755-758 (2016).

780 39. Gross, L. A., Baird, G. S., Hoffman, R. C., Baldridge, K. K. \& Tsien, R. Y. The structure 781 of the chromophore within DsRed, a red fluorescent protein from coral. Proc. Natl. Acad. Sci. U. S. A. 97, 11990-11995 (2000).

783 40. Heim, R., Cubitt, A. B. \& Tsien, R. Y. Improved green fluorescence. Nature 373, 663-664 784 (1995).

785 41. Kabsch, W. Integration, scaling, space-group assignment and post-refinement. Acta Crystallogr. Sect. D Biol. Crystallogr. 66, 133-144 (2010).

787 42. McCoy, A. J. Solving structures of protein complexes by molecular replacement with Phaser. Acta Crystallogr. Sect. D Biol. Crystallogr. 63, 32-41 (2006). 
43. Ai, H. W., Henderson, J. N., Remington, S. J. \& Campbell, R. E. Directed evolution of a monomeric, bright and photostable version of Clavularia cyan fluorescent protein: Structural characterization and applications in fluorescence imaging. Biochem. J. 400, $531-540$ (2006).

793

44. Emsley, P. \& Cowtan, K. Coot: Model-building tools for molecular graphics. Acta Crystallogr. Sect. D Biol. Crystallogr. 60, 2126-2132 (2004).

45. Adams, P. D. et al. PHENIX: A comprehensive Python-based system for macromolecular structure solution. Acta Crystallogr. Sect. D Biol. Crystallogr. 66, 213-221 (2010).

46. Strong, M. et al. Toward the structural genomics of complexes: Crystal structure of a PE/PPE protein complex from Mycobacterium tuberculosis. Proc. Natl. Acad. Sci. U. S. A. 103, 8060-8065 (2006).

47. Schrodinger LLC. The PyMOL Molecular Graphics System, Version 1.8. (2015).

801 48. Pettersen, E. F. et al. UCSF Chimera - A visualization system for exploratory research and analysis. J. Comput. Chem. 25, 1605-1612 (2004).

49. Wallace, A. C., Laskowski, R. A. \& Thornton, J. M. LIGPLOT: a program to generate schematic diagrams of protein-ligand interactions. Protein Eng. 8, 127-134 (1995).

50. Hanwell, M. D. et al. Avogadro: an advanced semantic chemical editor, visualization, and analysis platform. J. Cheminform. 4, 17 (2012).

807 51. Vanquelef, E. et al. R.E.D. Server: A web service for deriving RESP and ESP charges and building force field libraries for new molecules and molecular fragments. Nucleic Acids Res. 39, 511-517 (2011).

52. Wink, L. H., Baker, D. L., Cole, J. A. \& Parrill, A. L. A benchmark study of loop modeling methods applied to G protein-coupled receptors. J. Comput. Aided. Mol. Des. 
33, 573-595 (2019).

813 53. Jarzynski, C. Nonequilibrium Equality for Free Energy Differences. Phys. Rev. Lett. 78,

$814 \quad 2690-2693(1997)$.

815 54. Roe, D. R. \& Cheatham, T. E. PTRAJ and CPPTRAJ: Software for processing and

816 analysis of molecular dynamics trajectory data. J. Chem. Theory Comput. 9, 3084-3095

817 (2013).

818 55. Miller, B. R. et al. MMPBSA.py: An efficient program for end-state free energy

819 calculations. J. Chem. Theory Comput. 8, 3314-3321 (2012).

820 56. Humphrey, W., Dalke, A. \& Schulten, K. VMD - Visual Molecular Dynamics. J. Mol.

$821 \quad$ Graph. 14, 33-38 (1996).

822 57. Kalderon, D., Roberts, B. L., Richardson, W. D. \& Smith, A. E. A short amino acid

823 sequence able to specify nuclear location. Cell 39, 499-509 (1984).

824 